\title{
Variation in dust properties in a dense filament of the Taurus molecular complex (L1506)
}

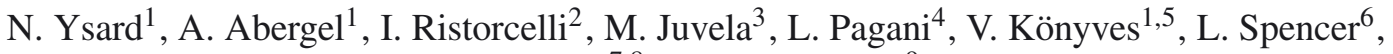 \\ G. White ${ }^{7,8}$, and A. Zavagno 9
}

\author{
${ }^{1}$ IAS, CNRS (UMR8617), Université Paris Sud, Bât. 121, 91400 Orsay, France \\ e-mail: nathalie.ysard@ias.u-psud.fr \\ 2 IRAP, CNRS (UMR5277), Université Paul Sabatier, 9 avenue du Colonel Roche, BP 44346, 31028 Toulouse Cedex 4, France \\ 3 Department of Physics, PO Box 64, University of Helsinki, 00014 Helsinki, Finland \\ ${ }^{4}$ LERMA, CNRS (UMR8112), Observatoire de Paris, 61 avenue de l'Observatoire, 75014 Paris, France \\ 5 Laboratoire AIM, CEA/DSM-CNRS-Université Paris Diderot, IRFU/Service d'Astrophysique, CEA Saclay, Orme des Meurisiers, \\ 91191 Gif-sur-Yvette, France \\ ${ }^{6}$ School of Physics and Astronomy, Cardiff University, Queens Buildings, The Parade, Cardiff CF24 3AA, UK \\ 7 The Open University, Department of Physics and Astronomy, Milton Keynes MK7 6AA, UK \\ 8 The Rutherford Appleton Laboratory, Chilton, Didcot, Oxfordshire OX11 0NL, UK \\ 9 Laboratoire d'Astrophysique de Marseille, CNRS/INSU Université de Provence, 13388 Marseille Cedex 13, France
}

Received 12 June 2013 / Accepted 5 September 2013

\section{ABSTRACT}

\begin{abstract}
Aims. We observed the L1506 filament, which is located in the Taurus molecular complex, with the Herschel PACS and SPIRE instruments. Our aim is to prove the variation in grain properties along the entire length of the filament. In particular, we want to determine above which gas density this variation arises and what changes in the grain optical properties/size distribution are required. Methods. We use the 3D radiative transfer code CRT, coupled to the dust emission and extinction code DustEM, to model the emission and extinction of the dense filament. We test a range of optical properties and size distributions for the grains: dust of the diffuse interstellar medium (interstellar PAHs and amorphous carbons and silicates) and both compact and fluffy aggregates.

Results. We find that the grain opacity has to increase across the filament to fit simultaneously the near-IR extinction and Herschel emission profiles of L1506. We interpret this change to be a consequence of the coagulation of dust grains to form fluffy aggregates. Grains similar to those in the diffuse medium have to be present in the outer layers of the cloud, whereas aggregates must prevail above gas densities of a few $10^{3} \mathrm{H} / \mathrm{cm}^{3}$. This corresponds to line-of-sights with visual extinction in the $V$ band of the order of 2 to 3. The dust opacity at $250 \mu \mathrm{m}$ is raised by a factor of 1.8 to 2.2 , while the grain average size is increased by a factor of 5 . These exact numbers depend naturally on the dust model chosen to fit the data. Our findings agree with the constraints given by the study of the gas molecular lines. Using a simple approach, we show that the aggregates may have time to form inside the filament within the cloud lifetime. Our model also characterises the density structure of the filament, showing that the filament width is not constant along L1506 but instead varies by a factor of the order of 4 .

Conclusions. We confirm the need for an increase in the far-IR dust opacity to explain the emission and extinction in L1506C, which we interpret as being due to dust growth. We also show that this opacity variation is valid along the entire length of the L1506 dense filament.
\end{abstract}

Key words. ISM: individual objects: L1506 - ISM: clouds - dust, extinction - evolution

\section{Introduction}

Recent observations have shown that prestellar cores preferentially form inside the dense molecular filaments observed both with molecular gas lines and the thermal dust farIR/submillimetre (submm) emission (Hartmann 2002; André et al. 2010; Men'shchikov et al. 2010; Hill et al. 2011; Nguyen Luong et al. 2011). It is thus important to characterise the physical properties of dust and gas in the filamentary structures of the interstellar medium (ISM) and to investigate how the starformation process depends on these initial conditions. Only with knowledge of these properties will we be able to derive reliable and quantitative information about the dense filaments such as the way they are formed, their masses, their structures, or their evolutionary stages (disrupting, collapsing, or fragmenting into cores). The dust grains are a key factor for the ISM evolution as they control, for example, the heating of the gas and the formation of $\mathrm{H}_{2}$ in the diffuse and dense ISM, respectively.

The dust emission in filaments has been thoroughly observed in a wide wavelength range during the past twenty years resulting in a few well-established facts. Firstly, it has been shown that the dust temperature decreases from the diffuse ISM $\left(n_{\mathrm{H}} \lesssim 100 \mathrm{H} / \mathrm{cm}^{3}\right)$ towards the centre of the dense molecular clouds, where $n_{\mathrm{H}} \gtrsim 10^{4}-10^{5} \mathrm{H} / \mathrm{cm}^{3}$, (Laureijs et al. 1991; Bernard et al. 1999) and that this decrease cannot be explained only by the extinction of the radiation field. Secondly, this decrease most often comes with a decrease in the mid- to far-IR dust emission ratio (Abergel et al. 1994, 1996; Bernard et al. 1999). Another striking fact is the joint increase in the observed dust opacity with density, which is preferentially observed at far-IR and submillimetre wavelengths (Cambrésy et al. 2001; Stepnik et al. 2003; Kramer et al. 2003; Flagey et al. 2009). 
This increase has been confirmed by a number of recent observations made with Planck, BLAST (Balloon Borne Large Aperture Submillimetre Telescope), and Herschel (Juvela et al. 2011; Planck Collaboration 2011a; Martin et al. 2012; Fischera \& Martin 2012b; Roy et al. 2013). They proved an increase of at least a factor of 2 at $250 \mu \mathrm{m}$ from the diffuse ISM to the centre of the molecular clouds, assuming that the dust emission is well approximated by a single modified blackbody. The study of Ysard et al. (2012) showed that such an increase cannot be due to radiative transfer effects but should originate in intrinsic variations in the dust properties. These aforementioned observational facts are usually attributed to grain growth occurring as a consequence of coagulation, which entails the disappearance of the smallest grains that emit in the mid-IR (Ossenkopf \& Henning 1994; Stognienko et al. 1995; Köhler et al. 2011, 2012). Coagulation produces larger grains on average with higher absorption cross-sections at long wavelengths, which explains both the lower temperatures and the increased opacity. If they manage to become large enough $(a \sim 0.5-1 \mu \mathrm{m})$, these grains are also expected to scatter light efficiently in the mid-IR (i.e., coreshine). This has recently been observed first in L183 and subsequently towards many molecular clouds (Pagani et al. 2010b; Steinacker et al. 2010; Stutz et al. 2010).

In this paper, we study a cloud in the Taurus molecular complex, L1506, which has been observed with the ESA Herschel Space Observatory (Pilbratt et al. 2010). The dust emission at one location across this filament (L1506C, $\alpha_{2000}=4 \mathrm{~h} 18 \mathrm{~m} 50 \mathrm{~s}$, $\left.\delta_{2000}=+25^{\circ} 19^{\prime} 43.6^{\prime \prime}\right)$ was previously modelled in detail by Stepnik et al. (2003) using IRAS (InfraRed Astronomical Satellite) and PRONAOS balloon-borne experiment observations (PROgramme NAtional d'AstrOnomie Submillimétrique). This analysis provided direct observational evidence for the first time of a significant increase in the dust opacity in the densest part of the cloud compared to the diffuse surrounding medium. They concluded that the dust opacity had to be multiplied by a factor of $3.4_{-0.7}^{+0.3}$ above a density threshold of $n_{\mathrm{H}}=(3 \pm 1) \times 10^{3} \mathrm{~cm}^{-3}$ (corresponding to $A_{V}=2.1 \pm 0.5^{\mathrm{m}}$ ). This was interpreted as a result of the formation of fluffy dust aggregates via coagulation processes, as predicted by dust model calculations (Ossenkopf \& Henning 1994; Ossenkopf 1993; Bazell \& Dwek 1990; Wright 1987). However, an increase of 3.4 seems difficult to explain from a theoretical point of view. For instance, Köhler et al. (2012) demonstrated that aggregates made of amorphous silicates and carbons can hardly reach an opacity enhancement of 2.7 when 16 grains are stuck together, which already leads to very long coagulation timescales. To better understand the physical properties of L1506C, molecular gas emission lines $\left({ }^{13} \mathrm{CO}, \mathrm{C}^{18} \mathrm{O}, \mathrm{N}_{2} \mathrm{H}^{+}\right)$were later observed and modelled by Pagani et al. (2010a). Explaining the molecular line profiles requires a central density at least seven times larger than the density inferred by Stepnik et al. (2003). This discrepancy comes mostly from the low angular resolution of the PRONAOS data $\left(\sim 4^{\prime}\right)$. Furthermore, Pagani et al. (2010a) observed a very strong CO depletion inside L1506C, up to a factor of 30 (lower limit) in the densest part of the filament, and an extremely low turbulence with an upper limit of $v_{\text {FWHM }} \sim 68 \mathrm{~m} / \mathrm{s}$. They also showed the evidence of a collapsing core detached from its envelope, which would be the signature of a prestellar core in the making. Thus, L1506C appears to be very interesting because of both variations in dust properties and its key evolutionary stage. Finally, because L1506C is one of the most cited examples to illustrate grain growth in dense clouds, we revisit the dust emission analysis. We take advantage of the angular resolution of the Herschel data, which is ten times higher than the resolution of
PRONAOS data, and of the constraints given by the molecular gas study (Pagani et al. 2010a). We also expand our analysis to the full length of L1506, instead of considering just L1506C. Our aim is to check whether the dust optical properties have actually evolved in L1506 and if so, at which density and by how much.

The paper is organised as follows. In Sect. 2, we describe the Herschel observations and data reduction. In Sect. 3, we explain how we produced visual extinction maps from the 2MASS data. In Sect. 4, we present the models we use to calculate the dust emission, extinction, and the radiative transfer through the filament. In Sect. 5, we detail the methods that are used to simultaneously fit the dust emission in the five Herschel spectral bands and how the best-fitting models are compared to extinction. In Sect. 6, we give the results from modelling L1506 and then discuss them in Sect. 7. We give our conclusions in Sect. 8.

\section{Herschel observations and data reduction}

Within the framework of the two Herschel key programmes, Evolution of interstellar dust (Abergel et al. 2010) and the Herschel Gould Belt survey (André et al. 2010), the Taurus S3 filament (L1506) has been mapped by the ESA Herschel Space Observatory with the SPIRE (Spectral and Photometric Imaging Receiver) and PACS (Photodetector Array Camera and Spectrometer) instruments (Griffin et al. 2010; Poglitsch et al. 2010). The observations were conducted on September 19, 2010 in the parallel-mode with fast, $60^{\prime \prime} / \mathrm{s}$, scanning speed. Two perpendicular maps were observed both with PACS (70 and $160 \mu \mathrm{m})$ and SPIRE $(250,350$, and $500 \mu \mathrm{m})$ of a commonly covered $100^{\prime} \times 60^{\prime}$ field for a total observing time of $2.3 \mathrm{~h}$.

The SPIRE maps reported in this paper are the Level 2 naive maps delivered by the Herschel Science Centre (HIPE version 7.0.1991) with standard corrections for instrumental effects and glitches. Striping induced by offsets in the flux calibration from one detector to another was removed using the Scan Map Destriper module included in the HIPE environment. The overall absolute flux accuracy is dominated by the calibration uncertainty and is conservatively estimated to be $\pm 7 \%$.

The PACS data were processed with the map-maker SANEPIC (Signal and Noise Estimation Procedure Including Correlations), as described in Patanchon et al. (2008). The overall absolute flux accuracy is dominated by the calibration uncertainty and is conservatively estimated to be $\pm 20 \%$.

The maps have an angular resolution of 8.4, 13.5, 18.2, 24.9, and $36.3^{\prime \prime}$ at $70,160,250,350$, and $500 \mu \mathrm{m}$, respectively. The maps were all convolved to the angular resolution of the $500 \mu \mathrm{m}$ SPIRE band of $36.3^{\prime \prime}$ (0.02 pc at $\left.140 \mathrm{pc}\right)$, assuming Gaussian beams. The maps used in this paper are presented in Fig. 1.

\section{Extinction from 2 MASS data}

The extinction map of the L1506 filament is computed with the NICER method (Lombardi \& Alves 2001). We use the Two Micron All Sky Survey (2MASS) data (Skrutskie et al. 2006) from which measurements of low photometric reliability were first removed $(S / N<5$ or $\sigma>0.217 \mathrm{mag}$ ). The sigma-clipping algorithm described in Lombardi \& Alves (2001) is used with the default threshold of $3 \sigma$. Because the maximum $A_{V}$ is only of about 10 to 20 magnitudes in L1506, we omit extinctiondependent colour corrections of the 2MASS data, since their effect would be only at the level of one percent (Roy et al. 2013). The calculations also require an assumption for the shape of the 

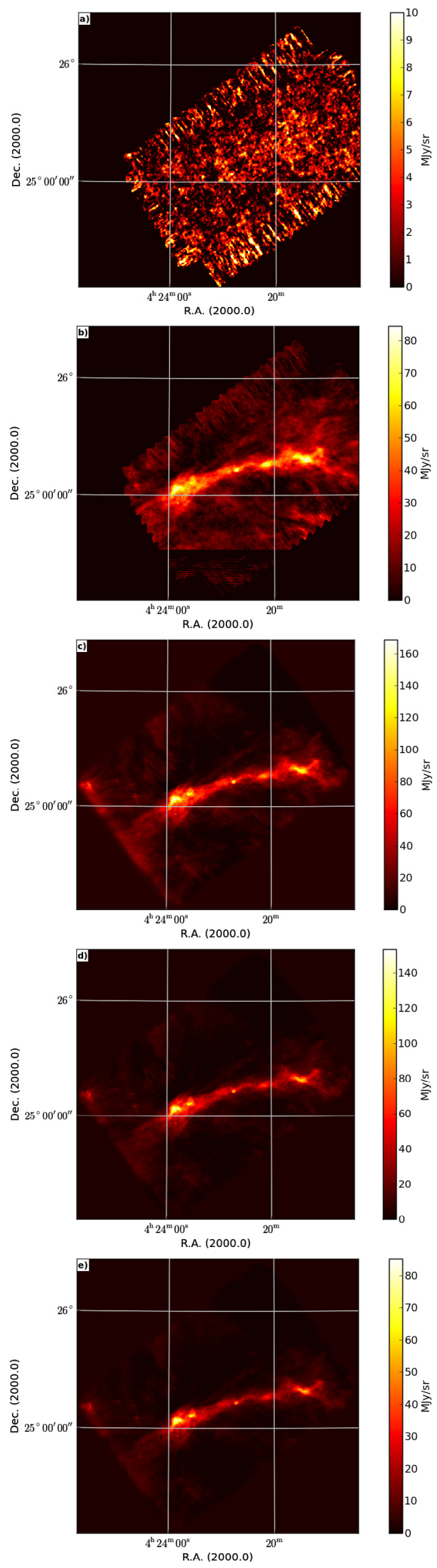

Fig. 1. Brightness maps of the Taurus filament at $70 \mu \mathrm{m}$ a); $160 \mu \mathrm{m} \mathrm{b}$ ); $250 \mu \mathrm{m} \mathrm{c)} ; 350 \mu \mathrm{m} \mathrm{d})$; and $500 \mu \mathrm{m} \mathrm{e)}$. extinction curve of the dust grains. This curve is usually described with the parameter $R_{V}=A_{V} / E(B-V)$, which is expected to vary from $R_{V} \sim 3.1$ in the diffuse medium to $R_{V} \sim 5.5$ or even higher in dense molecular clouds (Cardelli et al. 1989; Fitzpatrick 1999). The changes are however small in the near-IR domain (Cardelli et al. 1989) and should not have a significant effect on results expressed as near-IR extinction ${ }^{1}$. However, a change from $R_{V}=3.1$ to $R_{V}=5.5$ corresponds to a $\sim 14 \%$ drop in the values expressed in $A_{V}$. For this reason, we computed two different extinction maps: one with $R_{V}=3.1$ and the other with $R_{V}=5.5$, assuming that the extinction curve follows the prescription of Cardelli et al. (1989). These two maps are calculated at a spatial resolution of $F W H M=200^{\prime \prime}$ (i.e., 3.3'). In the case of a varying column density within individual cells, the NICER estimates can be biased towards low values (Juvela et al. 2008). As shown in Appendix A, this bias is of the order of $25 \%$ for the densest parts of the filament and reaches $\sim 15 \%$ for the less dense ones.

\section{Models: Shape of the cloud and dust properties}

As the dust emission profiles are affected simultaneously by the radiative transfer and the dust properties, we use the coupling of the 3D Monte-Carlo radiative transfer code called CRT (Continuum Radiative Transfer, Juvela 2005) and the dust emission and extinction code DustEM (Compiègne et al. 2011; Ysard et al. 2012). The DustEM code is used to determine the dust temperature distribution and emission. It is a versatile code that works with any kind of dust properties and size distributions.

\subsection{Shape of the cloud}

As described in Juvela (2005) and Ysard et al. (2012), CRT is a 3D Monte-Carlo radiative transfer code that allows for three types of cloud geometry: spheres, cylinders, and threedimension clouds defined on a Cartesian grid.

As can be seen in Fig. 1, L1506 seems to have a filamentary structure. However, this may be the result of its projection onto the plane of the sky; alternatively, it could be a sheet, projected edge-on. To model the emission lines of ${ }^{13} \mathrm{CO}, \mathrm{C}^{18} \mathrm{O}$, and $\mathrm{N}_{2} \mathrm{H}^{+}$, Pagani et al. (2010a) showed that $\mathrm{L} 1506 \mathrm{C}$ must have a central $\mathrm{H}_{2}$ density of $20000 \lesssim n\left(\mathrm{H}_{2}\right) \lesssim 50000 \mathrm{~cm}^{-3}$. This leads to a peak column density of about $8 \times 10^{21} \lesssim N\left(\mathrm{H}_{2}\right) \lesssim 2 \times 10^{22} \mathrm{H}_{2} / \mathrm{cm}^{2}$ for a spherical model, which is similar to the results of Goldsmith et al. (2008) for the entire length of L1506. The ratio of the column density to the local density gives a rough estimate of the depth of the cloud in the direction perpendicular to the plane of the sky, which is about $0.05 \lesssim N\left(\mathrm{H}_{2}\right) / n\left(\mathrm{H}_{2}\right) \lesssim 0.32 \mathrm{pc}$. This depth is comparable to the width of the cloud projected onto the plane of the sky, which is about $3^{\prime}$ to $5^{\prime}$ along the filament, equivalent to 0.12 to $0.3 \mathrm{pc}$ at a distance of $140 \mathrm{pc}$. This width is also much smaller than the total length of L1506, which is about $2.5 \mathrm{pc}$. We conclude it is a good approximation to model L1506 as a cylinder-like filament.

Many observational studies have shown that the density distribution of molecular clouds appears to be flat at their centres and then decreases following a power-law at larger radii (Ward-Thompson et al. 1994; Bergin \& Tafalla 2007). Recently, Herschel data were used to characterise this power-law in the case of filamentary clouds. For instance, Arzoumanian et al. (2011) found that the 27 filaments observed in the IC 5146

1 Recent work started to question this issue but without giving physical explanation for an $E(J-K)$ variation (van Breemen et al. 2011). 


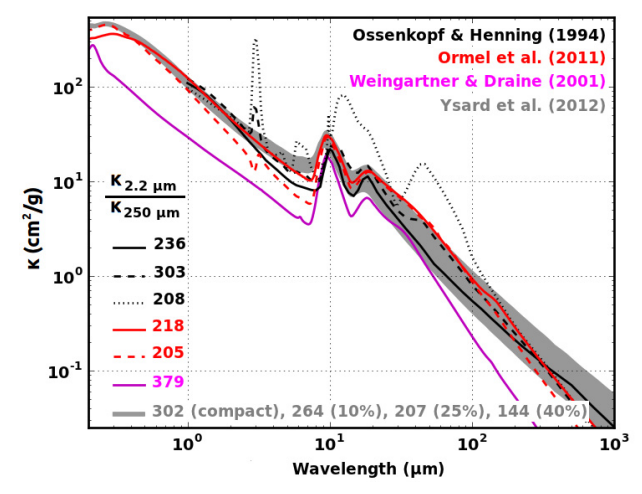

Fig. 2. Opacity for the aggregate populations used in this paper (grey area). The magenta line shows the opacity of the dust model "A" of Weingartner \& Draine (2001). We also display the opacity of the bare aggregates (black line) and of the aggregates covered by a thin (black dashed line) or thick (black dotted line) ice mantle described in Ossenkopf \& Henning (1994). The red lines show the aggregate opacities computed by Ormel et al. (2011) without (solid line, their model sil, gra) and with an ice mantle (dashed line, their model ic-sil, ic-gra). The numbers on the left side of the figure give the near- to far-IR opacity ratio for these dust models.

molecular cloud have density profiles $\sim r^{-2 \pm 0.5}$. A similar result was found by Palmeirim et al. (2013) in the Taurus molecular complex for the dense filaments B211 and L1495 and by Juvela et al. (2012) for 24 filaments identified as Galactic cold clumps with Planck HFI data (Planck Collaboration 2011d). As a result, we represent the density distribution of L1506 with the following Plummer-like function:

$n_{\mathrm{H}}(r)=\frac{n_{\mathrm{C}}}{1+\left(r / R_{\mathrm{flat}}\right)^{2}}$,

where $n_{\mathrm{C}}$ is the central density and $R_{\text {flat }}$ is the central "flat" radius under which the density varies little. Except where otherwise stated, all the modelled filaments are assumed to lay in the plane of the sky with their axis of symmetry along L1506.

\subsection{Dust optical properties}

For the dust optical properties, we consider two of the cases studied by Ysard et al. (2012). First, we consider a population of grains typical of the diffuse ISM as defined by Compiègne et al. (2011): the DHGL populations (Dust at High Galactic Latitude). The DHGL populations consist of interstellar polycyclic aromatic hydrocarbons (PAHs) small and large amorphous carbons (SamC and LamC, respectively), and astronomical silicates (aSil). For the small grains (PAHs \& SamC), we use log-normal size distributions with central radii $a_{0}$ and widths $\sigma$. For the larger grains (LamC \& aSil), we use a power-law distribution $a^{\alpha}$, starting at $a=4 \mathrm{~nm}$, with an exponential cut-off $\mathrm{e}^{-\left[\left(a-a_{t}\right) / a_{c}\right]^{2}}$ for $a \geq a_{t}$ (Weingartner \& Draine 2001; Compiègne et al. 2011). The dust model abundances and the parameters of the size distributions are given in Table 1.

Second, we consider a population of "evolved" grains ${ }^{2}$. These grains consist of aggregates, which are a mixture of small and large grains, with both carbons and silicates. Aggregates are expected to form in dense molecular clouds, possibly with large fractions of voids or porosity (Dorschner \& Henning 1995). Their optical properties are calculated using Mie theory combined with the effective medium theory (EMT) using

\footnotetext{
2 The motivation behind the dust models described in this section is discussed further in Ysard et al. (2012).
}

Table 1. Dust model abundances and size distribution parameters (see Sect. 4.2 for details).

\begin{tabular}{|c|c|c|c|c|c|c|}
\hline \multicolumn{7}{|c|}{ Small grains (DHGL) } \\
\hline & $\begin{array}{c}\rho \\
\left(\mathrm{g} / \mathrm{cm}^{3}\right)\end{array}$ & $\sigma$ & $\begin{array}{c}a_{0} \\
(\mathrm{~nm})\end{array}$ & $\begin{array}{c}Y \\
\left(M_{\text {dust }} / M_{\mathrm{H}}\right)\end{array}$ & $\begin{array}{c}\kappa_{250 \mu \mathrm{m}} \\
\left(\mathrm{cm}^{2} / \mathrm{g}\right)\end{array}$ & $\beta$ \\
\hline PAH & 2.24 & 0.40 & 0.64 & $7.80 \times 10^{-4}$ & 0.001 & \\
\hline SamC & 1.81 & 0.35 & 2.00 & $1.65 \times 10^{-4}$ & 0.002 & 1.55 \\
\hline \multicolumn{7}{|c|}{ Big grains (DHGL) } \\
\hline & $\rho$ & $\alpha$ & $\begin{array}{l}a_{c}, a_{t} \\
(\mathrm{~nm})\end{array}$ & $Y$ & $\kappa_{250 \mu \mathrm{m}}$ & $\beta$ \\
\hline LamC & 1.81 & -2.8 & 150.0 & $1.45 \times 10^{-3}$ & 0.014 & 1.55 \\
\hline aSil & 3.5 & -3.4 & 200.0 & $7.8 \times 10^{-3}$ & 0.034 & 2.11 \\
\hline \multicolumn{7}{|c|}{ Aggregates } \\
\hline & $\rho$ & $\alpha$ & $a_{c}, a_{t}$ & Y & $\kappa_{250 \mu \mathrm{m}}$ & $\beta$ \\
\hline $0 \%$ & 2.87 & -2.4 & 234.0 & $1.02 \times 10^{-2}$ & 0.111 & 1.33 \\
\hline $10 \%$ & 2.59 & -2.4 & 242.0 & $1.02 \times 10^{-2}$ & 0.140 & 1.32 \\
\hline $25 \%$ & 2.16 & -2.4 & 256.0 & $1.02 \times 10^{-2}$ & 0.208 & 1.30 \\
\hline $40 \%$ & 1.72 & -2.4 & 276.0 & $1.02 \times 10^{-2}$ & 0.331 & 1.27 \\
\hline
\end{tabular}

Notes. Parameter $\rho$ is the grain mass density, $Y$ is the mass abundance per $\mathrm{H}, \kappa_{250} \mu \mathrm{m}$ is the opacity at $250 \mu \mathrm{m}$, and $\beta$ is the intrinsic opacity spectral index for $\lambda>100 \mu \mathrm{m}$ for each dust population. For aggregates, the percentages correspond to the volume of voids they contain, which indicates how porous they are.

the Bruggeman rule (Bohren \& Huffman 1983). As in Ysard et al. (2012), we consider compact aggregates and porous aggregates with $10 \%, 25 \%$, and $40 \%$ of voids. The dust model abundances and the parameters of the size distributions are presented in Table 1. Through aggregation, the dust opacity is increased by a factor equal to the ratio of the absorption efficiency of the aggregates to the mean absorption efficiency of isolated DHGL carbon and silicate grains of the same volume. This corresponds to an increase of 1.84 for compact aggregates, 2.16 for porous aggregates with $10 \%$ of voids, 2.84 for $25 \%$, and 3.89 for $40 \%$. These aggregates have a low opacity spectral index in the farIR and the submm of $\beta \sim 1.3$, resulting from the shape of the absorption efficiency of the large grains of the DHGL populations (see also Sect. 7.3). The aggregate opacities are displayed in Fig. 2. For comparison, the aggregate dust models, as obtained after $10^{5}$ yrs of coagulation, of Ormel et al. (2011) and Ossenkopf \& Henning (1994) for $n_{\mathrm{H}}=10^{6} \mathrm{H} / \mathrm{cm}^{3}$ are shown in this figure. We also show the Milky Way dust model "A" of Weingartner \& Draine (2001), which is not composed of aggregates but of diffuse-ISM type grains (Li \& Draine 2001) with a modified size distribution to allow for bigger grains. This model was normalized according to Draine (2003) and corresponds to $R_{V}=5.5$. We note that the opacity of our model of aggregates is close to those developed by Ormel et al. (2011) and Ossenkopf \& Henning (1994). The main difference between these models is their spectral index in the far-IR and submm.

\section{Modelling and fitting methods}

In this section, we describe how we subdivide the observed irregular filament into four separate and homogeneous filamentary pieces to probe the dust properties at various locations along L1506. We also detail our fitting method and the free parameters considered. 

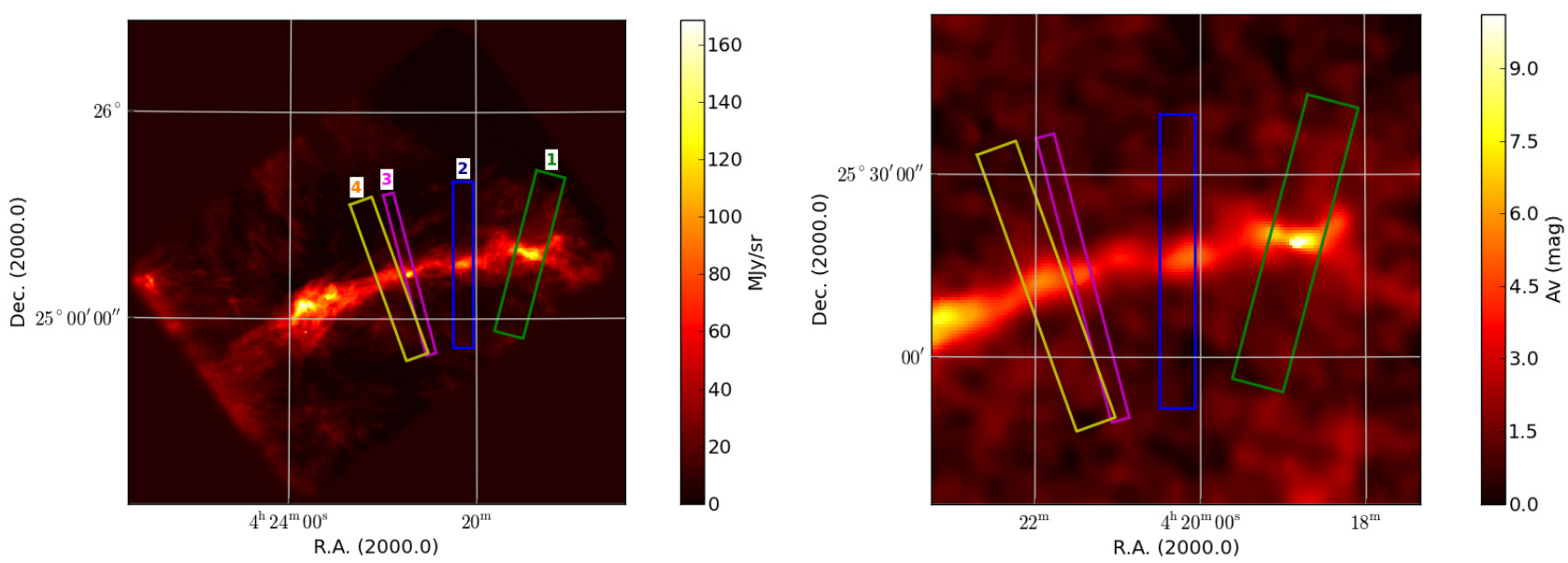

Fig. 3. Left: brightness map of the filament at $250 \mu \mathrm{m}$. The rectangles show the positions of the four cuts of Table 2 (green is the first one, blue the second, magenta the third, and yellow the fourth). Right: extinction map $A_{V}$ at $0.55 \mu \mathrm{m}$ calculated from 2MASS data for $R_{V}=3.1$.

Table 2. Coordinates of the centres and widths of the cuts across the L1506 filament.

\begin{tabular}{cccc}
\hline \hline & RA (J2000) & Dec $(\mathrm{J} 2000)$ & Width \\
\hline Cut 1 & $4 \mathrm{~h} 18 \mathrm{~m} 51 \mathrm{~s}$ & $+25 \mathrm{~d} 18 \mathrm{~m} 43.6 \mathrm{~s}$ & $8.6^{\prime} / 0.35 \mathrm{pc}$ \\
Cut 2 & $4 \mathrm{~h} 20 \mathrm{~m} 16.7 \mathrm{~s}$ & $+25 \mathrm{~d} 15 \mathrm{~m} 46.3 \mathrm{~s}$ & $3.0^{\prime} / 0.12 \mathrm{pc}$ \\
Cut 3 & $4 \mathrm{~h} 21 \mathrm{~m} 25.7 \mathrm{~s}$ & $+25 \mathrm{~d} 13 \mathrm{~m} 2.5 \mathrm{~s}$ & $5.8^{\prime} / 0.24 \mathrm{pc}$ \\
Cut 4 & $4 \mathrm{~h} 21 \mathrm{~m} 53.2 \mathrm{~s}$ & $+25 \mathrm{~d} 11 \mathrm{~m} 41 \mathrm{~s}$ & $8.2^{\prime} / 0.33 \mathrm{pc}$ \\
\hline
\end{tabular}

\subsection{Filament description}

We selected four locations along the filament that we want to model (Table 2 and Fig. 3). The first location, or first cut across the filament, corresponds to the filament modelled by Stepnik et al. (2003) and Pagani et al. (2010a) that they named L1506C (green box in Fig. 3). The second and the fourth cuts cross less dense areas, while the third one is located on a brighter and more compact spot (blue, yellow, and magenta boxes in Fig. 3, respectively). To define the brightness profiles corresponding to one location, we use a box perpendicular to the filament, the width of which is chosen so that the brightness along the filament remains approximately constant (Fig. 3). Then, the brightness profile is defined as the average value of each line of pixels parallel to the filament axis. The error bars are defined as the quadratic addition of the standard deviation of each line of pixels plus $7 \%$ of the brightness profile in the case of SPIRE bands and $20 \%$ in the case of PACS bands to account for the calibration uncertainties (see Sect. 2). The corresponding profiles are shown in Fig. 4 for the emission and the visual extinction. We note that the resolution of 36.3" allows us to resolve the filament.

As we are mostly interested in the densest component of the filament where the gas is predominantly molecular, we separate the filament into two parts: a diffuse envelope and the actual dense molecular filament. The diffuse envelope is fitted with a second order polynomial as in Stepnik et al. (2003) for offsets of 0.5 to $1 \mathrm{pc}$ from the centre of the cut coordinates given in Table 2. The envelopes in emission and extinction are shown in Figs. 4. The actual brightness and extinction profiles that we fit in the following sections are what remains when the polynomials are removed from the data. Using this subtraction has also the advantage of defining a zero-level common to all the SPIRE and PACS channels and to the extinction map.

\subsection{Fitting method}

For each part of the filament, our aim is to simultaneously fit the five Herschel brightness profiles and the 2MASS extinction profile. For a given grain type (size distribution and optical properties), the parameters to be fitted are the incident radiation field, the outer radius of the filament $R_{\text {out }}$, its flat central radius $R_{\text {flat }}$, and its central density $n_{\mathrm{C}}$. For the grains, we use the properties described in Sect. 4.2, either mixed or not. Planck Collaboration (2011a) computed the dust grain temperature in the Taurus molecular complex, performing modified blackbody fits of the Planck-HFI and IRAS data $(100 \mu \mathrm{m}$ to $2 \mathrm{~mm})$. In the diffuse medium surrounding the dense filaments, the thermal dust temperature ranges between $\sim 16$ to $20 \mathrm{~K}$. These values are close to the value measured in the standard diffuse ISM (Planck Collaboration $2011 \mathrm{~b}, \mathrm{c})$. The radiation field illuminating the L1506 filament is thus assumed to be close to the standard radiation field, hereafter addressed as the ISRF (Draine \& Anderson 1985). As a result, we test several radiation fields: the ISRF and this same ISRF extinguished and reddened by a layer of DHGL grains with $A_{V}^{\text {ext }}=0.05,0.1,0.2,0.3,0.4,0.5,0.6,0.7,0.8,0.9$, and $1^{\mathrm{m}}$ (corresponding roughly to $0.9 \gtrsim G_{0} \gtrsim 0.3$ ). For $R_{\text {out }}$, we test values between 0.15 and $0.5 \mathrm{pc}$, and we try 30 different values of $R_{\text {flat }}$ between $R_{\text {out }} / 200$ and $R_{\text {out }} / 1.2$, which correspond to steps of $0.005 \mathrm{pc}$.

For each run, we take one set of those values (grain type, $A_{V}^{\text {ext }}, R_{\text {out }}$, and $R_{\text {flat }}$ ), and the free parameter is the central density $n_{\mathrm{C}}$ that we vary to minimize the $\chi^{2}$ for the fit between the Herschel and the modelled brightness profiles. Starting from the smallest $\chi^{2}$, we consider all the models for which the modelled profiles are within the error bars for the five Herschel bands. These models are then compared to the extinction profile calculated with the 2MASS data either for $R_{V}=3.1$ or 5.5 (see Sect. 3). For each model, if the modelled visual extinction at the centre of the modelled filament does not match the 2MASS $A_{V}$ value, the model is discarded. The uncertainties on the derived physical parameters that are quoted in the following section reflect the size of the family of models, which provide a good fit to both the emission and the extinction profiles.

\section{Dust emission and extinction modelling}

We now apply the methods described in the previous section to the four cuts given in Table 2 . 

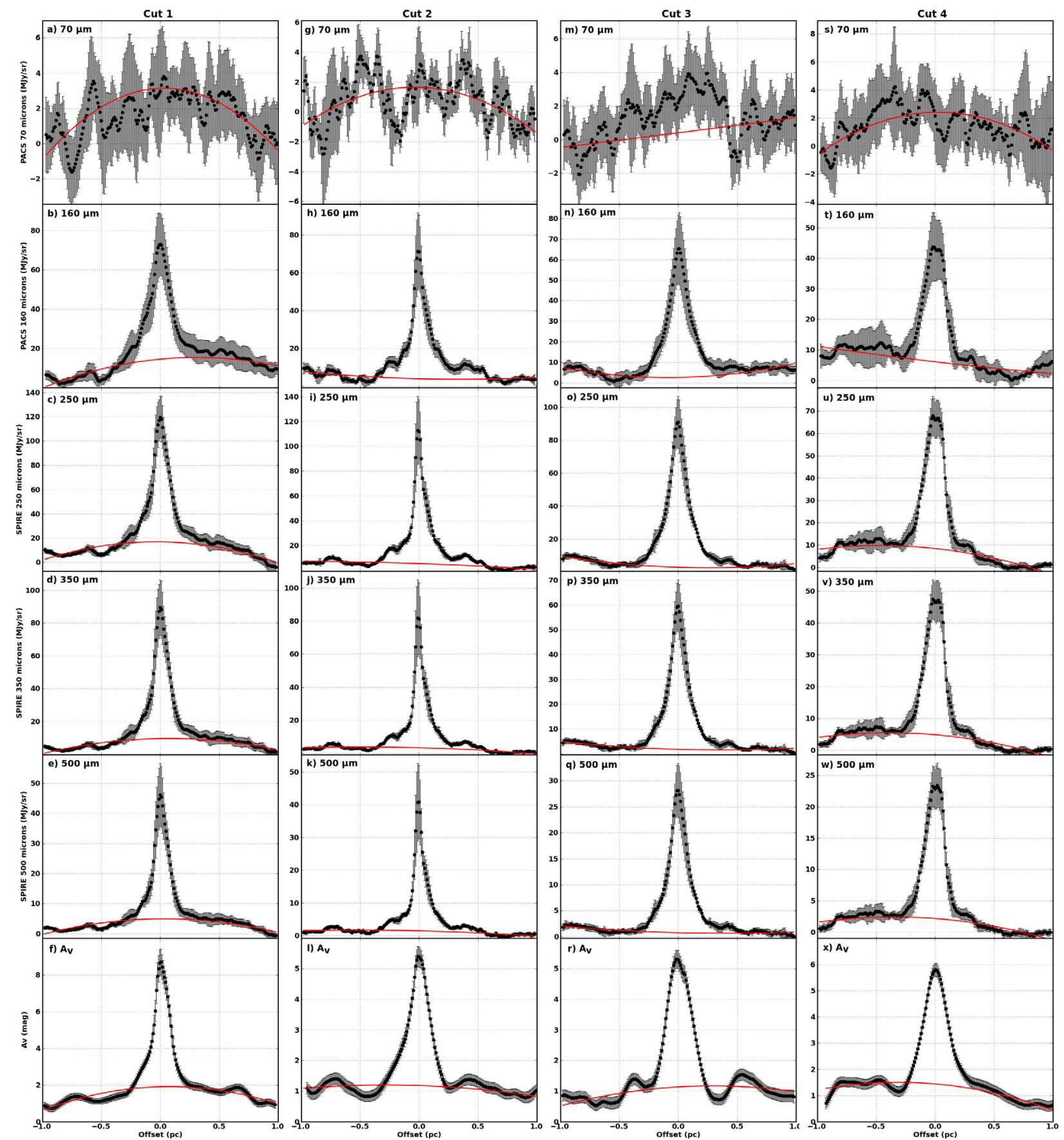

Fig. 4. Emission brightness profiles of the first cut (see Table 2) at $70 \mu \mathrm{m}$ a); $160 \mu \mathrm{m} \mathrm{b}$ ); $250 \mu \mathrm{m}$ c); $350 \mu \mathrm{m} \mathrm{d}$ ); and $500 \mu \mathrm{m}$ e); and extinction profile calculated from the 2 MASS data with $R_{V}=3.1 \mathbf{f}$ ). The same for the second cut $\mathbf{g}$ ) $-\mathbf{l}$ ); the third cut $\left.\mathbf{m}\right)-\mathbf{r}$ ); and the fourth cut $\mathbf{s}$ ) $-\mathbf{x}$ ). The red lines show the second order polynomials fitted to remove the relatively diffuse envelope from the densest filament.

In all of the cases presented in this section, the best fits were obtained for the standard ISRF, so we do not present results obtained for attenuated radiation fields. Furthermore, the best fits were obtained for the same outer radius, irrespective of which kind of grains were considered. Consequently, we only show the results for those $R_{\text {out }}$ values, which are 0.3 pc for the first cut, $0.4 \mathrm{pc}$ for the second cut, $0.32 \mathrm{pc}$ for the third cut, and $0.2 \mathrm{pc}$ for the fourth cut.

\subsection{DHGL}

First, we assume that the grain size distribution and optical properties in the dense filament are the same as the diffuse ISM. To mimic that, we attempt to fit the brightness profiles with cylinders filled with DHGL populations (see Sect. 4.2). The bestfitting models are shown in Fig. 5, where it can be seen that the brightness profiles cannot be reproduced with DHGL populations for cut 1,2 , and 3 . The emission profiles corresponding to the best-fitting models are within the error bars of the five Herschel emission profiles for only cut 4 (Figs. 5s-w), which, as can be seen in Fig. 3, has the lowest density of any of the cuts. However, the extinction profile corresponding to this best fit model is a factor of 6.1 higher than the 2MASS extinction profile calculated for $R_{V}=3.1$, the typical value for dust in the diffuse medium (Figs. 5f, 1, r, x). Factors of 4.4, 5.5, and 3.8 are found for cuts 1,2 , and 3 . Indeed, the fitting of the 

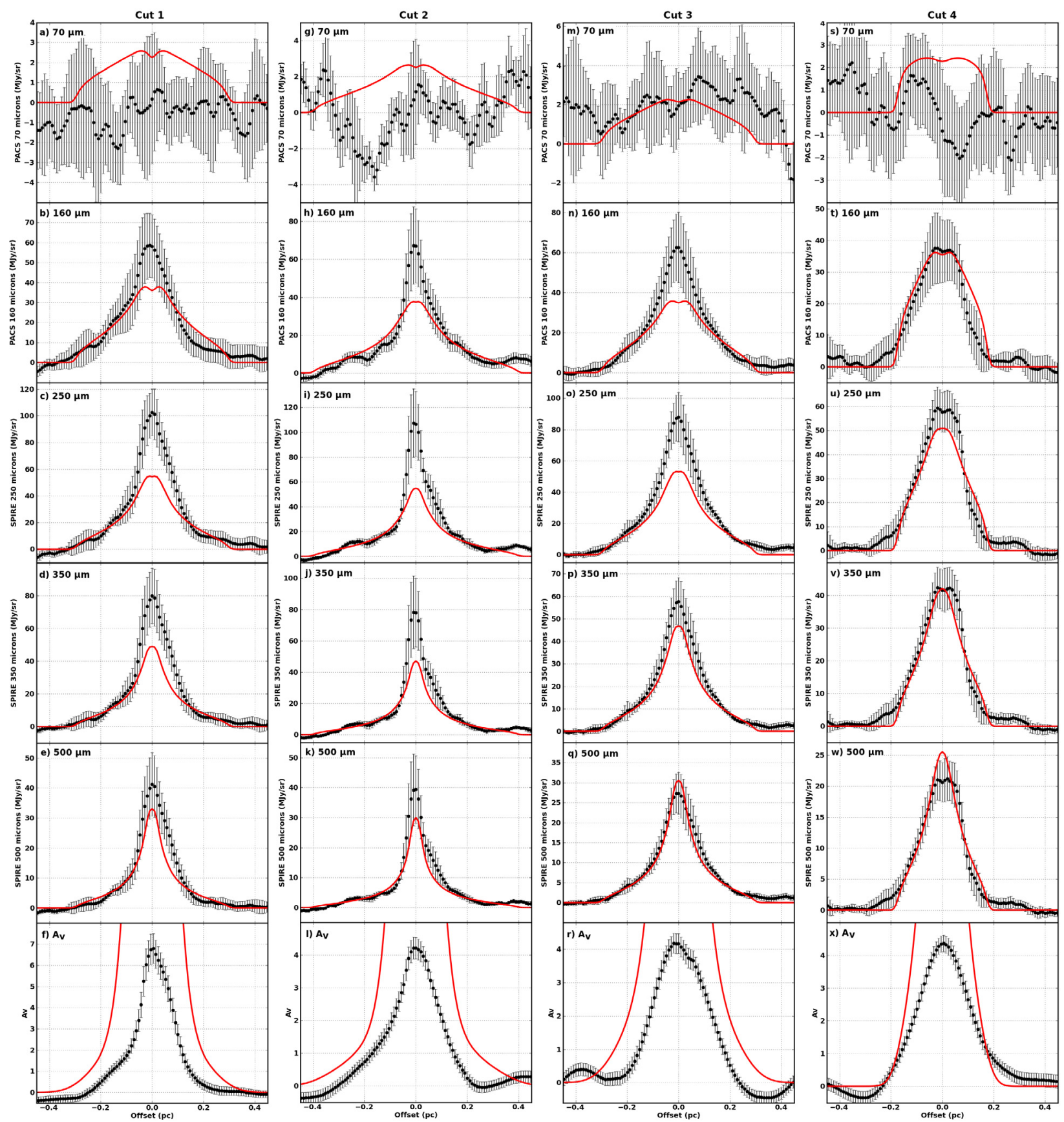

Fig. 5. The red lines show the best fits with DHGL grains of the emission brightness profiles (background removed) for the first cut at $70 \mu \mathrm{m}$ a); $160 \mu \mathrm{m} \mathrm{b}) ; 250 \mu \mathrm{m} \mathrm{c}) ; 350 \mu \mathrm{m} \mathrm{d}$ ); and $500 \mu \mathrm{m} \mathbf{e})$; and the corresponding visual extinction profile f). In the last row, the black dots show the extinction profile calculated from 2MASS data with $R_{V}=3.1$. The same are shown for the second cut $\mathbf{g}$ ) $-\mathbf{l}$ ); the third cut $\mathbf{m}$ ) $-\mathbf{r}$ ); and the fourth cut s)-x).

brightness profiles requires high gas densities for the grains to be cold enough not to emit too much in the PACS $70 \mu \mathrm{m}$ spectral band, leading to extinction values always higher than the 2MASS extinction. That means that the grain optical properties are not the same as the diffuse ISM. The grains have to evolve from the diffuse to the dense medium; this also applies to the relative abundance of the small grains (both large (LamC, aSil) and small grains (SamC) contribute to the emission at $70 \mu \mathrm{m}$ ).

To ascertain that this result does not depend on the choice of dust model, we repeated the fitting using the dust properties defined by Draine \& Li (2007) for the diffuse ISM. This model is made of a mixture of amorphous silicates, graphite, and interstellar PAHs. Using this second dust model, we find similar results; that is, it is not possible to fit all the emission and extinction profiles simultaneously. We also considered the case of modelled cylinders with inclination angles of $10,20,30$, and $40^{\circ}$, which did not permit to fit the data either. Consequently, we definitely exclude the possibility that the dust in L1506 is similar to that found in the diffuse ISM.

\subsection{Aggregates}

As it appears that the grain properties have to vary from the diffuse surroundings of the filament to its centre, we model cylinders filled only with aggregates, which range from compact to 

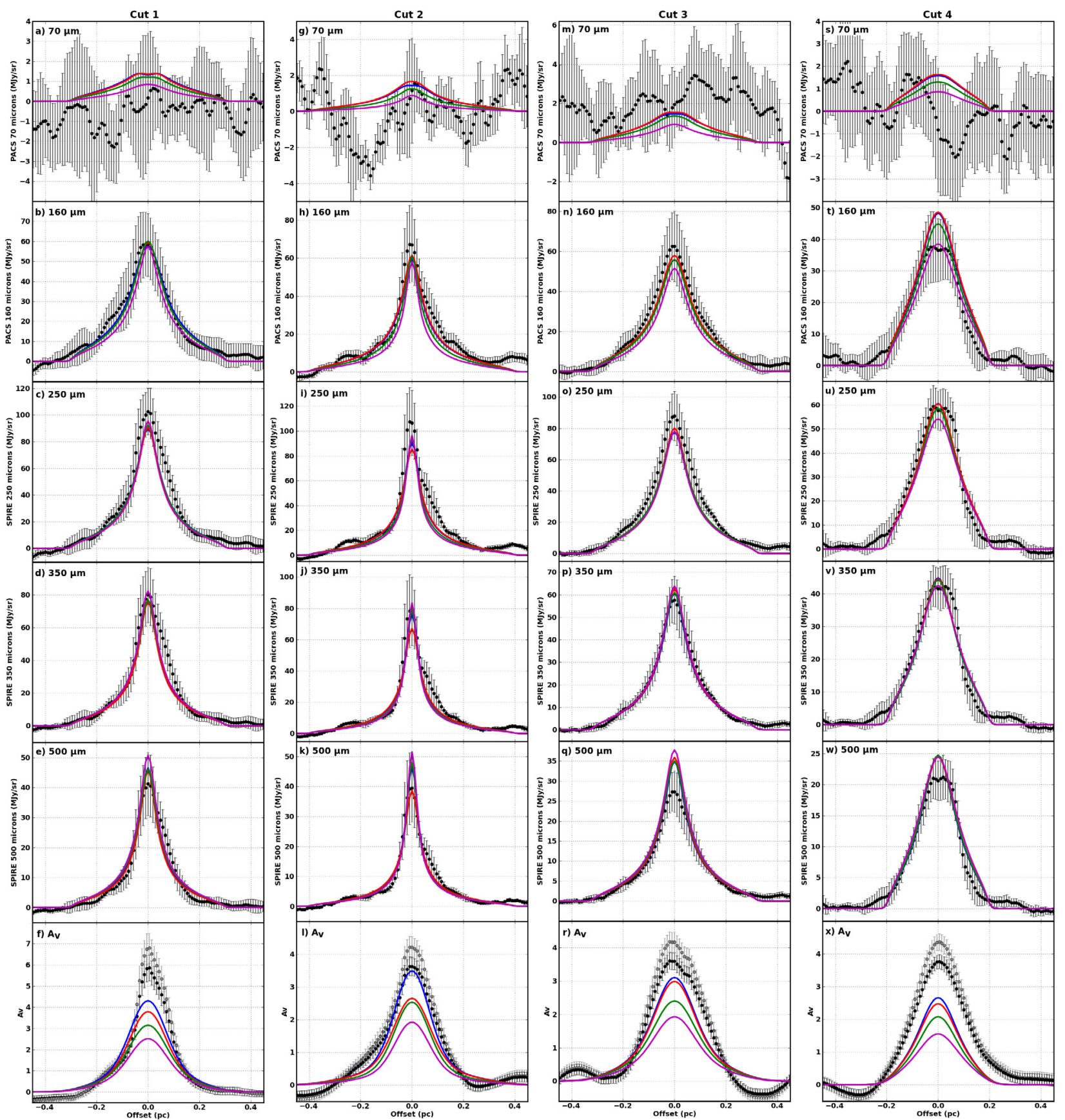

Fig. 6. The lines show the best fits with aggregates of the emission brightness profiles for the first cut at $70 \mu \mathrm{m}$ a); $160 \mu \mathrm{m} \mathrm{b);250} \mu \mathrm{m}$ c); $350 \mu \mathrm{m}$ d); $500 \mu \mathrm{m} \mathrm{e}$ ); and the corresponding extinction profile $\mathbf{f}$ ). The grey dots show the extinction profile calculated from $2 \mathrm{MASS}$ with $R_{V}=3.1$ and the black ones with $R_{V}=5.5$. The same are shown for the second cut $\mathbf{g}$ ) $-\mathbf{l}$ ); the third cut $\mathbf{m}$ ) $-\mathbf{r}$ ); and the fourth cut $\left.\left.\mathbf{s}\right)-\mathbf{x}\right)$. The blue lines are for compact aggregates, the red lines for aggregates with $10 \%$ of voids, the green lines with $25 \%$ of voids, and the magenta lines with $40 \%$ of voids.

porous grains containing 10,25 , or $40 \%$ of voids. These aggregates are a mixture of all the dust populations of the DHGL model (Compiègne et al. 2011). In this case, we assume that all the grains are already evolved inside the filament, which no longer contains small grains, since they have already been incorporated into the aggregates. The best-fitting models are displayed in Fig. 6. The five brightness profiles of cuts 1, 2, and 4 can be well reproduced with those aggregates. For cut 3 , the SPIRE $500 \mu \mathrm{m}$ brightness profile cannot be fitted by any of the aggregates tested here. Our best-fitting model for this cut leads to an overestimate of the emission at $500 \mu \mathrm{m}$ by a factor of 1.3-1.4 depending on the aggregate type. In Figs. 6f, 1, r, x, the extinction profiles corresponding to these best-fitting models are compared to the 2MASS extinction profiles calculated with $R_{V}=3.1$ and 5.5. Only the extinction profile of the second cut is well fitted by a cylinder filled with compact aggregates. For the other cuts, as the aggregates cool efficiently, the fits of the submillimetre emission require densities that are too low to explain the 2MASS visual extinction by factors of 1.5 to $2.5,1.3$ to 2.0, and 1.5 to 2.6 for cut 1,3 , and 4 , respectively, and for compact to porous aggregates with $40 \%$ of voids, respectively. Thus, considering a filament with an inclination angle of $10,20,30$, or $40^{\circ}$ does not permit to fit the data either. This means that for at least three of the four cuts the grain properties have to vary inside the dense molecular part of the filament. 


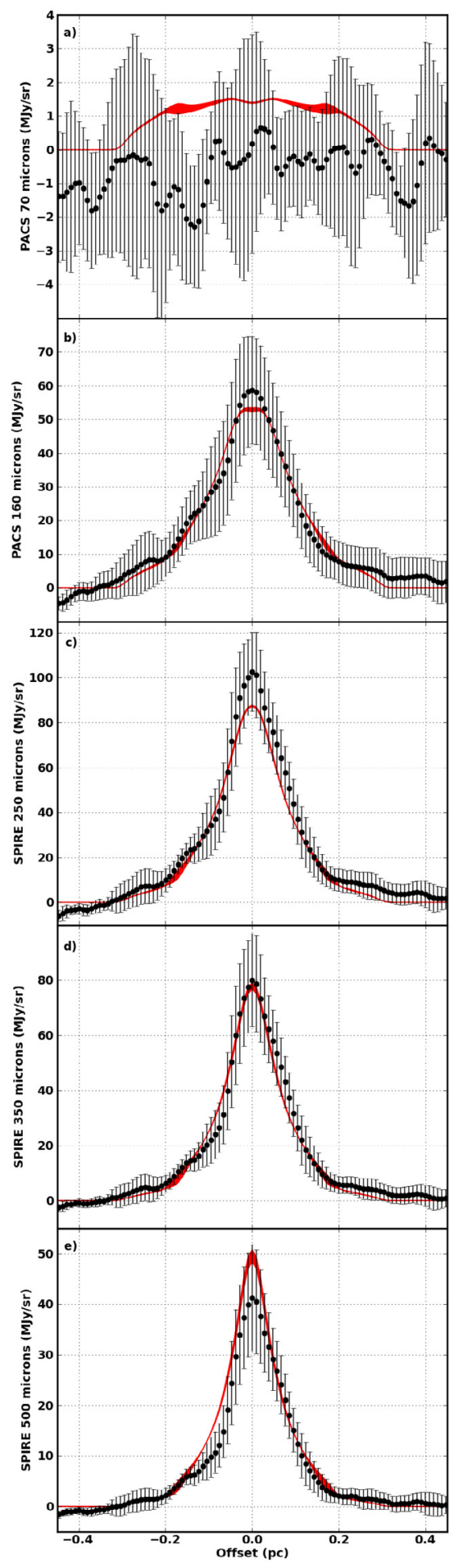

Fig. 7. Brightness profiles of the first cut at 70, 160, 250, 350, and $500 \mu \mathrm{m}$ (panels a); b); c); d); and e) respectively). The red areas are the best-fitting models for porous aggregates with $10 \%$ of voids and $n_{\mathrm{T}}=1500 \pm 250 \mathrm{H} / \mathrm{cm}^{3}$.

\subsection{Evolution across the filament}

The results of the previous sections indicate that the grain properties need to vary as a function of position within the filament, especially in its dense centre. In this case, we assume that DHGL populations are present in the outer layers and that the grain properties no longer correspond to DHGL but instead to aggregates above a given threshold density. We choose to represent

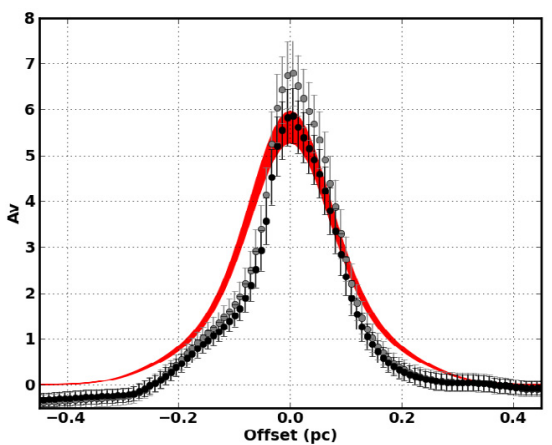

Fig. 8. Extinction profile of the first cut calculated from 2MASS data with $R_{V}=3.1$ (grey dots) and $R_{V}=5.5$ (black dots). The red area corresponds to the best-fitting models presented in Fig. 7.

this threshold by a step function to keep a reasonable number of free parameters. Thus, the value of the threshold density simply tells above which density the aggregates dominate the dust mass. This threshold density, $n_{\mathrm{T}}$, is a new parameter and we test $n_{\mathrm{T}}=125,250,375,500,625,750,875,1000,1125$, $1250,1375,1500,1750,2250,2500,3000,3500,4000,4500$, 5000, 5500, 6000, 6500, 7000, 7500, 8000, 8500, 9000, 9500, and $10000 \mathrm{H} / \mathrm{cm}^{3}$. The fitting method remains the same: for a given parameter combination [grain type, $A_{V}^{\mathrm{ext}}, R_{\text {out }}, R_{\text {flat }}, n_{\mathrm{T}}$ ], the free parameter to minimize the $\chi^{2}$ is still the central density $n_{\mathrm{C}}$. The parameters of the best-fitting models are given in Table 3 and described in the following sections.

\subsubsection{First cut (L1506C)}

The results for the first cut are shown in Figs. 7 and 8. The molecular gas lines in L1506C have been studied in detail by Pagani et al. (2010a) and yield a strong constraint on the central density $n_{\mathrm{C}}$. To explain the emission lines of $\mathrm{N}_{2} \mathrm{H}^{+}, \mathrm{C}^{18} \mathrm{O}$, ${ }^{13} \mathrm{CO}$, and $\mathrm{C}^{17} \mathrm{O}$, they found that $40000 \lesssim n_{\mathrm{C}} \lesssim 100000 \mathrm{H} / \mathrm{cm}^{3}$. The brightness and extinction profiles can be fitted simultaneously with compact aggregates for $n_{\mathrm{T}}=875 \pm 125 \mathrm{H} / \mathrm{cm}^{3}$ or with porous aggregates with $10 \%$ of voids for $n_{\mathrm{T}}=1500 \pm$ $250 \mathrm{H} / \mathrm{cm}^{3}$. However, in the case of compact aggregates the best-fitting models demand central densities between 150000 and $160000 \mathrm{H} / \mathrm{cm}^{3}$. These values are too high when compared with the results of Pagani et al. (2010a), so we exclude compact aggregates. In the case of porous aggregates with $10 \%$ of voids, the central densities are $90000 \lesssim n_{\mathrm{C}} \lesssim 110000 \mathrm{H} / \mathrm{cm}^{3}$, which agrees with the densities required by the model explaining the gas emission lines. Therefore, as written previously, our best-fitting model requires the aggregates with $10 \%$ of voids to prevail for $n_{\mathrm{T}}=1500 \pm 250 \mathrm{H} / \mathrm{cm}^{3}$, which corresponds to an offset of $0.19 \pm 0.03 \mathrm{pc}$ from the centre of the filament. It also corresponds to line-of-sights with a total $A_{V} \sim 2.0-3.2$ (without envelope subtraction, Fig. 4f). This value roughly matches the threshold value of $A_{V}=3.3 \pm 0.1$ for the detection of ice in the Taurus dark clouds measured by Whittet et al. (1988). Our threshold for the predominance of the aggregates coincides with the beginning of the decrease in the ${ }^{13} \mathrm{CO}$ abundance observed by Pagani et al. (2010a) between 0.15 and 0.23 pc. The desorption of volatile ice mantles can happen after cosmic-ray impacts (Roberts et al. 2007). However, this desorption is less efficient when the molecules have frozen-out onto the surface of large grains, the temperature of which is more stable while undergoing cosmic-ray impacts. Thus, the match between the start of the depletion of $\mathrm{CO}$ and the presence of large aggregates seems 


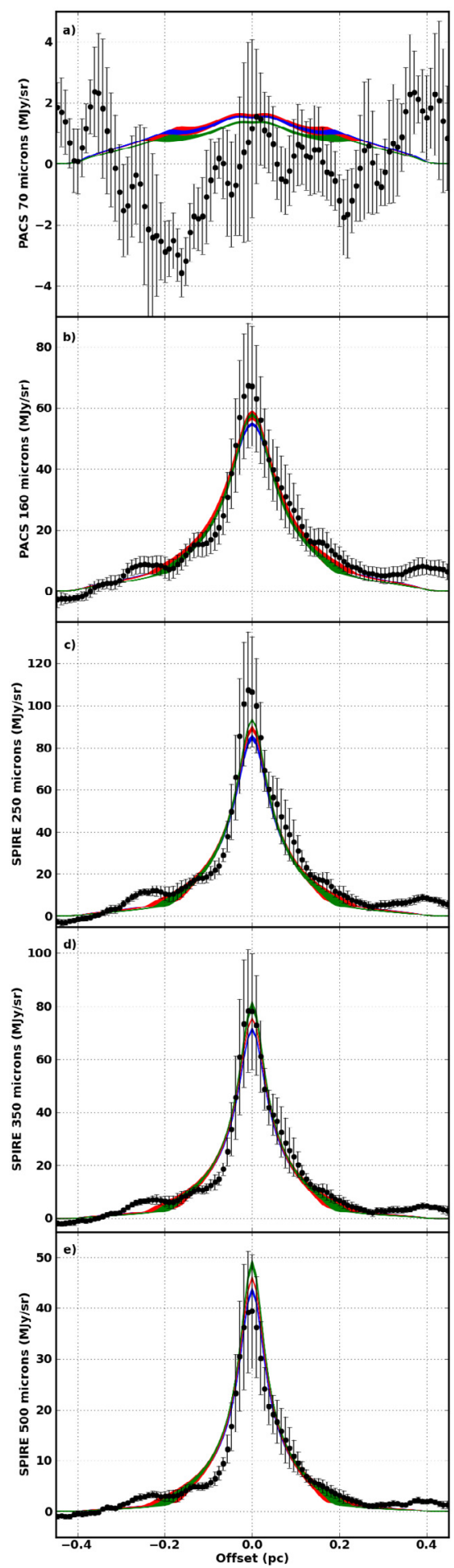

Fig. 9. Brightness profiles of the second cut at 70, 160, 250, 350, and $500 \mu \mathrm{m}$ (panels a); b); c); d); and e) respectively). The blue areas are the best-fitting models for compact aggregates and $n_{\mathrm{T}}=875 \pm 125 \mathrm{H} / \mathrm{cm}^{3}$, the red ones for porous aggregates with $10 \%$ of voids and $n_{\mathrm{T}}=750 \pm$ $250 \mathrm{H} / \mathrm{cm}^{3}$, and the green ones for porous aggregates with $25 \%$ of voids and $n_{\mathrm{T}}=625 \pm 125 \mathrm{H} / \mathrm{cm}^{3}$.

plausible. Furthermore, the presence of ice mantles on top of the grains is expected to increase the formation efficiency of the aggregates (Ormel et al. 2009).

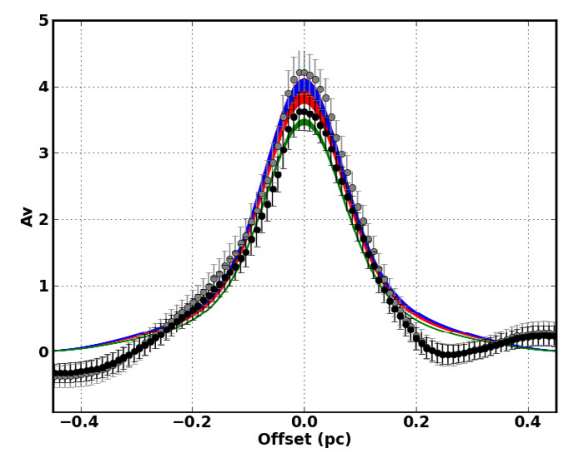

Fig. 10. Extinction profile of the second cut calculated from 2MASS data with $R_{V}=3.1$ (grey dots) and $R_{V}=5.5$ (black dots). The blue, red, and green areas correspond to the best-fitting models presented in Fig. 9.

\subsubsection{Second and fourth cuts}

The results for the second cut are presented in Figs. 9 and 10. Its emission and extinction profiles can be jointly fitted with compact aggregates for $n_{\mathrm{T}}=875 \pm 125 \mathrm{H} / \mathrm{cm}^{3}$, porous aggregates with $10 \%$ of voids for $n_{\mathrm{T}}=750 \pm 250 \mathrm{H} / \mathrm{cm}^{3}$, or porous aggregates with $25 \%$ of voids for $n_{\mathrm{T}}=625 \pm 125 \mathrm{H} / \mathrm{cm}^{3}$, which correspond to an offset of $0.12 \pm 0.02 \mathrm{pc}$ or line-of-sight with total $A_{V} \sim 2.0-3.6$. The central and threshold densities required to model this cut are similar to what is needed for cut 1 (L1506C).

The results for the fourth cut are shown in Figs. 11 and 12. The best-fitting models are obtained with compact aggregates for $n_{\mathrm{T}}=6000 \pm 500 \mathrm{H} / \mathrm{cm}^{3}$ or porous aggregates with $10 \%$ of voids for $n_{\mathrm{T}}=5500 \pm 250 \mathrm{H} / \mathrm{cm}^{3}$ in both cases. In cut 4 , the central density is about ten times lower than in cuts 1 and 2 with $n_{\mathrm{C}} \sim$ $12000 \mathrm{H} / \mathrm{cm}^{3}$, and the threshold density for the aggregates to be dominant is about 5 times higher. Thus, the aggregates seem to be present only at the very centre of the filament for offsets smaller than $0.08 \pm 0.01$ pc equivalent to total $A_{V} \geqslant 3.1-5.2$.

For these two cuts, the lack of constraints on the central density of the gas does not allow us to infer which kind of aggregates best reproduces the data. However, we notice that for the second and the fourth cuts the aggregates with $10 \%$ of voids, or similarly with an increase in the opacity at $250 \mu \mathrm{m}$ by a factor of 2.16, can explain the emission and extinction profiles as for the first cut (L1506C).

\subsubsection{Third cut}

Finally, we could not find any satisfactory fit for the third cut: the modelled cylinders always lead to an overestimate of the emission in the SPIRE $500 \mu \mathrm{m}$ band by a factor of 1.3-1.4 for our best-fitting model. To check that this caveat does not come from our earlier assumption of a filament in the plane of the sky, we tested four cylinder inclinations: $10^{\circ}, 20^{\circ}, 30^{\circ}$, and $40^{\circ}$. The results remain unchanged with an overestimate of the emission at $500 \mu \mathrm{m}$. We also notice that the best-fitting models for the three other cuts predict $500 \mu \mathrm{m}$ emission that is invariably in the upper values allowed by the error bars (Figs. 7e, 9e, and 11e). This means that the optical properties of our aggregates may have an opacity spectral index that is too low in the far-IR and the submm $(\beta \sim 1.3$, see Table 1 and Sect. 7.3). As we cannot derive physical parameters for this cut, it is excluded from the subsequent discussion. 
Table 3. Parameters of the best-fitting models for cuts 1,2 , and 4 , described in Sect. 6.3 , where $R_{\text {out }}$ is the external radius of the filament and the percentages in the "aggregates" lines give the percentage of voids in terms of grain volume.

\begin{tabular}{lcccccc}
\hline \hline & Cut 1 & \multicolumn{3}{c}{ Cut 2} & & Cut 4 \\
\hline$R_{\text {out }}(\mathrm{pc})$ & 0.30 & & 0.40 & 0.20 \\
Aggregates & $10 \%$ & $0 \%$ & $10 \%$ & $25 \%$ & $0 \%$ & $10 \%$ \\
$\kappa_{250 \mu \mathrm{m}}$ & $\times 2.16$ & $\times 1.84$ & $\times 2.16$ & $\times 2.84$ & $\times 1.84$ & $\times 2.16$ \\
$n_{\mathrm{C}}\left(\mathrm{H} / \mathrm{cm}^{3}\right)$ & $101800_{-3200}^{+9600}$ & $126300 \pm 2500$ & $121900_{-6400}^{+2000}$ & $96500 \pm 200$ & $12700_{-700}^{+600}$ & $11700 \pm 300$ \\
$R_{\text {flat }}(\mathrm{pc})$ & 0.0213 & 0.0152 & 0.0152 & 0.0152 & 0.0967 & 0.0967 \\
$n_{\mathrm{T}}\left(\mathrm{H} / \mathrm{cm}^{3}\right)$ & $1500 \pm 250$ & $875 \pm 125$ & $750 \pm 250$ & $625 \pm 125$ & $6000 \pm 500$ & $5500 \pm 250$ \\
$M_{\text {line }}\left(M_{\odot} / \mathrm{pc}\right)$ & $18.3_{-0.8}^{+1.7}$ & $14.7 \pm 0.3$ & $14.2_{-0.7}^{+0.2}$ & $11.2 \pm 0.02$ & $15.3_{-0.9}^{+0.8}$ & $14.1 \pm 0.4$ \\
$F W H M(\mathrm{pc})$ & $0.069 \pm 0.005$ & & $0.051 \pm 0.003$ & & $0.23 \pm 0.01$ \\
\hline
\end{tabular}

Notes. The following lines give the factor by which the opacity at $250 \mu \mathrm{m}$ is increased, $n_{\mathrm{C}}$ is the central proton density, $R_{\text {flat }}$ is the central flat radius, $n_{\mathrm{T}}$ is the threshold density above which DHGL populations disappear in favour of aggregates, $M_{\text {line }}$ is the mass per unit length of the filament, and FWHM is the width at half maximum of the hydrogen column density profile.

\subsubsection{Temperature distribution}

Figure 13 presents the temperature distributions that correspond to the best-fitting models obtained for aggregates with $10 \%$ of voids $\left(\kappa_{250 \mu \mathrm{m}} \times 2.16\right)$ as a function of the radius for cuts 1,2 , and 4 . These temperatures are the equilibrium temperatures of the grains, averaged over the size distribution. The central temperatures of the second and the fourth cuts are $T_{\text {dust }}=11.3 \pm$ $0.3 \mathrm{~K}$ and $T_{\text {dust }}=13.6 \pm 0.2 \mathrm{~K}$, respectively. For the first cut, we find it is significantly lower with $T_{\text {dust }}=10.2 \pm 0.3 \mathrm{~K}$. The fact that this value is very close to the gas temperature of about $10 \mathrm{~K}$ used by Pagani et al. (2010a) at the centre of L1506C is probably coincidental. Indeed for gas densities lower than $10^{5} \mathrm{H} / \mathrm{cm}^{3}$, a mild dust-gas coupling is expected. In this case, the dust grains are usually colder than the gas by $2-3 \mathrm{~K}$, especially when the grain growth is taken into account because it further decreases the dust-gas coupling (Zhilkin et al. 2009; Juvela \& Ysard 2011; Nielbock et al. 2012). This indicates that the gas temperature in Pagani et al. (2010a) may have been slightly underestimated.

\subsection{Column density distribution}

Figure 14 shows the hydrogen column density distributions corresponding to the best-fitting models for cuts 1,2 , and 4 (see Sects. 6.3.1 and 6.3.2). Their full widths at half maximum are $F W H M=0.069 \pm 0.005,0.051 \pm 0.003$, and $0.23 \pm 0.01 \mathrm{pc}$ for cuts 1,2 , and 4 , reaching $N_{\mathrm{H}} \sim 1.9 \times 10^{22}, 1.5 \times 10^{22}$, and $0.8 \times 10^{22} \mathrm{H} / \mathrm{cm}^{2}$, respectively. The width of L1506 is of the same order of that found by Arzoumanian et al. (2011), who studied a large sample of 90 filaments in the IC 5146, Polaris, and Aquila regions. Averaging along the length of the filaments, they found an almost constant width of $0.10 \pm 0.03 \mathrm{pc}$. However, our analysis shows that the width is not constant along the filament and that it varies by a factor $\sim 4$ according to the local column density in agreement with Juvela et al. (2012). We find that the smaller widths correspond to the densest parts of L1506, which agrees with the idea of a gravitationally contracting cloud.

An anti-correlation between the FWHM and the central column density was found by Fischera \& Martin (2012a), who analysed analytically isothermal cylinders confined by an external pressure. The FWHM that we find for cuts 1 and 2 match their values for a self-gravitating cloud (they computed $F W H M \sim$ 0.05 pc for $N_{\mathrm{H}} \sim 2 \times 10^{22} \mathrm{H} / \mathrm{cm}^{2}$, see their Fig. 8). However, we find that the FWHM of the fourth cut is much higher than any of their analytical values. The fourth cut corresponds to the less dense area that we studied. It has a central density 10 times lower than in the other cuts and a central column density approximately
Table 4. Averaged sizes in nanometres, $\langle a\rangle$, and densities, $n_{\mathrm{dust}} / n_{\mathrm{H}}$, for the DHGL populations.

\begin{tabular}{lcccc}
\hline \hline Dust type & PAH & SamC & LamC & aSil \\
\hline$\langle a\rangle^{a}$ & 0.6 & 2 & 9 & 7 \\
$n_{\text {dust }} / n_{\mathrm{H}}$ & $5.1 \times 10^{-7}$ & $2.6 \times 10^{-9}$ & $2.0 \times 10^{-11}$ & $2.4 \times 10^{-10}$ \\
PAH & 804 & 563 & 478 & 485 \\
SamC & 563 & 299 & 200 & 209 \\
LamC & 478 & 200 & 89 & 99 \\
aSil & 485 & 209 & 99 & 110 \\
$10 \%$ & 463 & 182 & 68 & 79 \\
\hline
\end{tabular}

Notes. Lines 3 to 6 (PAH to aSil) of the table give the critical speed between the species in $\mathrm{m} / \mathrm{s}$ (see Eq. (2)). The last line gives $v_{\text {crit }}$ for collisions with an aggregate containing $10 \%$ of voids $(\langle a\rangle \sim 20 \mathrm{~nm})$. (a) These sizes correspond to the average size over the size distribution in terms of grain number. In terms of mass, the average sizes are of 0.7 and $3.5 \mathrm{~nm}$ for the PAHs and the SamC, respectively, the size distributions of which are log-normals. For the LamC and the aSil grains, for which the size distributions decrease as power-laws, they are 221 and $255 \mathrm{~nm}$, respectively.

twice as low. Heitsch (2013) modelled turbulent isothermal hydrostatic infinite cylinders and explored the effect of gas accretion on the cloud structures. They showed that at low column densities the FWHM and the central $N_{\mathrm{H}}$ are expected to be uncorrelated with $F W H M=0.2-0.3 \mathrm{pc}$, which is similar to the width of the fourth cut. For $N_{\mathrm{H}}>10^{22} \mathrm{H} / \mathrm{cm}^{2}$, their calculations show a decreasing FWHM with increasing $N_{\mathrm{H}}$, agreeing with the width of the first and second cuts.

\section{Discussion}

We investigate here the possibility for the aggregates to form inside the dense filament in terms of coagulation timescales using the parameters of the best-fitting models, as described in the previous section for cuts 1,2 , and 4 . We also consider the stability of the filament against gravitational collapse.

\subsection{Coagulation timescale}

As seen in Sect. 6, the dust optical properties must vary from the outer layers of the filament to its denser centre to explain its emission and extinction profiles. Following some rough assumptions, we now estimate whether the relatively low density of this filament $\left(n_{\mathrm{H}} \lesssim 10^{5} \mathrm{H} / \mathrm{cm}^{3}\right)$ allows for the formation of 


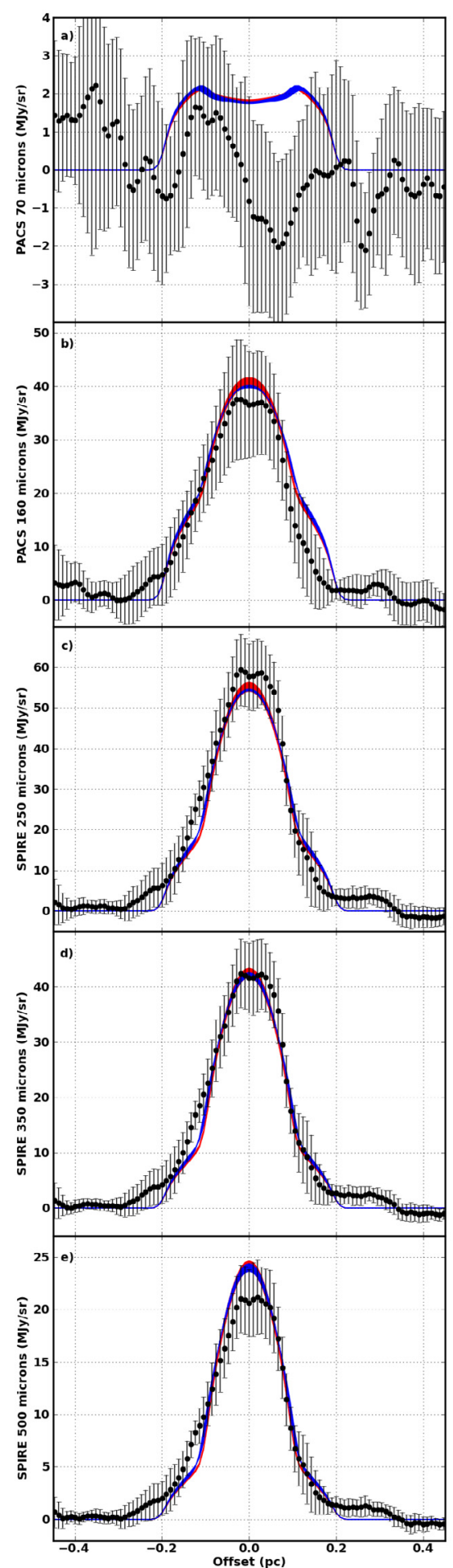

Fig. 11. Brightness profiles of the fourth cut at 70, 160, 250, 350, and $500 \mu \mathrm{m}$ (panels a); b); c); d); and e) respectively). The blue area is for the best-fitting models for compact aggregates and $n_{\mathrm{T}}=6000 \pm$ $500 \mathrm{H} / \mathrm{cm}^{3}$; the red one is for porous aggregates with $10 \%$ of voids and $n_{\mathrm{T}}=5500 \pm 250 \mathrm{H} / \mathrm{cm}^{3}$.

aggregates on a reasonable timescale. To answer this question, we use the simple approach described below.

The coagulation timescale depends on the grain densities, their sizes through the collisional cross-section, and the critical speed above which a collision would lead to destruction instead of aggregation. The average sizes and the densities of the four DHGL populations are listed in Table 4. According to

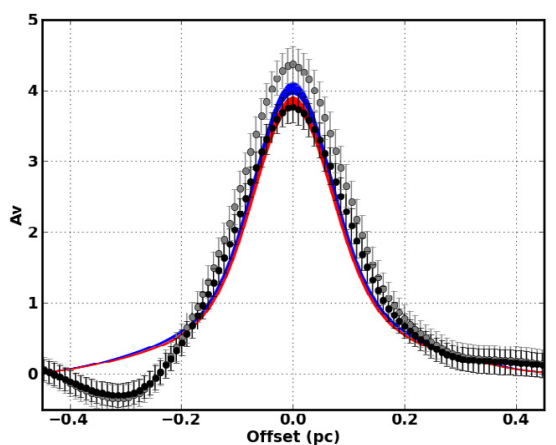

Fig. 12. Extinction profile of the fourth cut calculated from 2 MASS data with $R_{V}=3.1$ (grey dots) and $R_{V}=5.5$ (black dots). The blue and red areas correspond to the best-fitting models presented in Fig. 11.

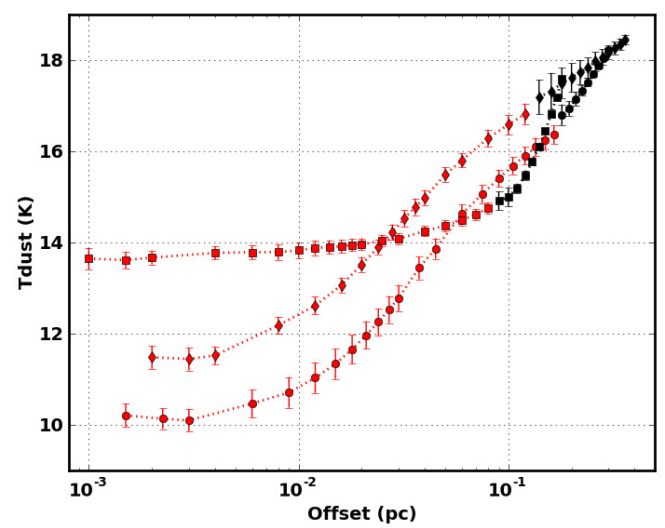

Fig. 13. Temperature distributions of the best-fitting models for the first (bullets), the second (diamonds), and the fourth cuts (squares). The red symbols are the equilibrium temperature of the aggregates with $10 \%$ of voids, averaged over their size distribution, and the black symbols are the same for the mixture of large grains of the DHGL populations (LamC and aSil).

Table 5. Coagulation timescales in years for cuts 1, 2, and 4.

\begin{tabular}{lccc}
\hline \hline & $\tau_{1}(\mathrm{yr})$ & $\tau_{2}(\mathrm{yr})$ & $\tau_{4}(\mathrm{yr})$ \\
\hline PAH on LamC & 70 & 60 & 580 \\
PAH on aSil & 80 & 60 & 660 \\
SamC on LamC & $8.5 \times 10^{3}$ & $7.1 \times 10^{3}$ & $7.3 \times 10^{4}$ \\
SamC on aSil & $9.6 \times 10^{3}$ & $8.0 \times 10^{3}$ & $8.3 \times 10^{4}$ \\
LamC on LamC & $4.4 \times 10^{5}$ & $3.7 \times 10^{5}$ & $3.8 \times 10^{6}$ \\
LamC on aSil & $4.9 \times 10^{5}$ & $4.1 \times 10^{5}$ & $4.3 \times 10^{6}$ \\
aSil on LamC & $4.0 \times 10^{4}$ & $3.4 \times 10^{4}$ & $3.5 \times 10^{5}$ \\
aSil on aSil & $4.5 \times 10^{4}$ & $3.7 \times 10^{4}$ & $3.9 \times 10^{5}$ \\
\hline
\end{tabular}

Notes. These are the coagulation timescales for the sticking of only two grains with a relative speed equal to the turbulent speed, $\Delta v=68 \mathrm{~m} / \mathrm{s}$.

Dominik \& Tielens (1997) and Stepnik et al. (2003), the critical speed for a destructive collision between two species 1 and 2 of sizes $a_{1}$ and $a_{2}$ can be expressed as

$v_{\text {crit }}=670\left[\left(\frac{1}{a_{1}}+\frac{1}{a_{2}}\right) 10^{-5} \mathrm{~cm}\right]^{5 / 6}(\mathrm{~cm} / \mathrm{s})$.

The critical speeds for collisions between species of the DHGL populations are given in Table 4 . Then, the coagulation timescale of two species depends on their cross-sections and densities, and on their relative velocity, $\Delta v$. Under most molecular cloud 


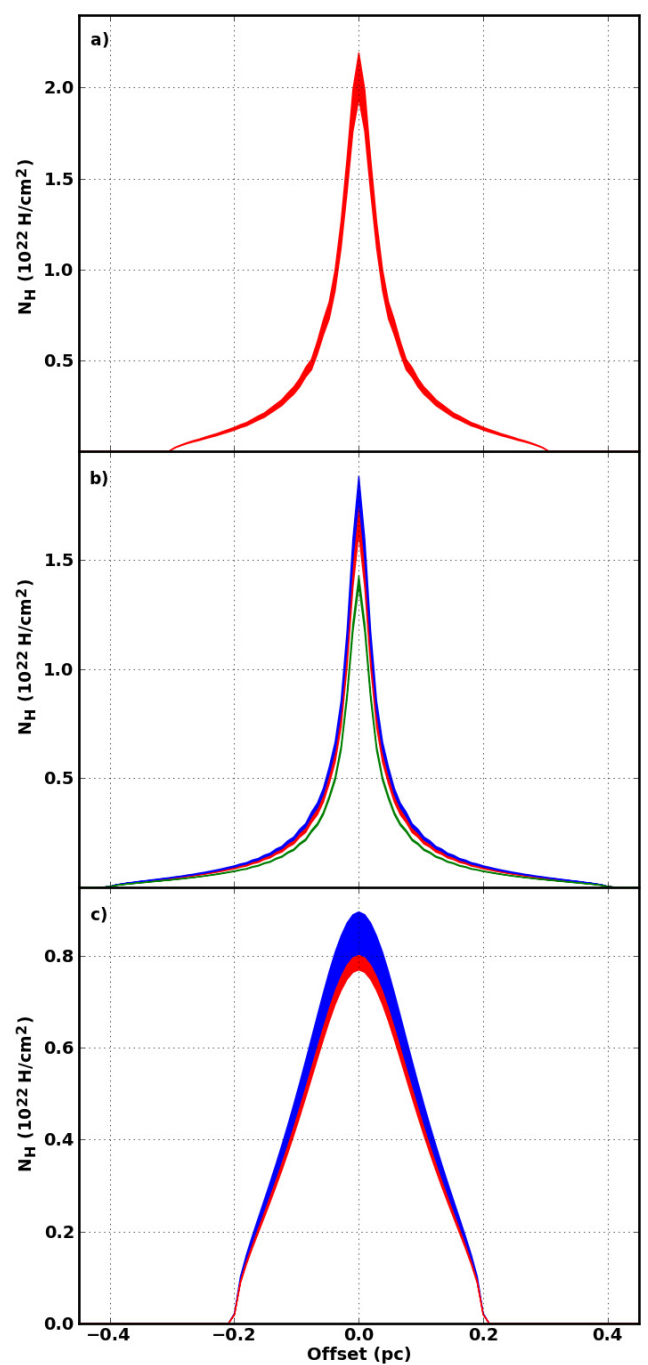

Fig. 14. Column density distributions of the best-fitting models for cuts $1 \mathbf{a}), 2 \mathbf{b}$ ), and $4 \mathbf{c}$ ), as a function of the offset from the filament centre. The blue areas show the best-fitting models using compact aggregates, the red areas using aggregates with $10 \%$ of voids, and the green areas with $25 \%$ of voids.

conditions, $\Delta v$ is defined by the turbulence (Ossenkopf 1993; Ormel \& Cuzzi 2007; Ormel et al. 2009). Because of its own inertia, a grain does not instantaneously follow the gas motion and as a result, the maximum velocity difference between two grains is the gas turbulent velocity. This means that in any case $\Delta v \leqslant v_{\text {turb }}$, where the actual velocity difference depends on the efficiency of the gas-dust coupling. In L1506C, the modelling of the $\mathrm{C}^{18} \mathrm{O}$ and $\mathrm{N}_{2} \mathrm{H}^{+}$lines at the centre of the cloud demonstrated a low turbulent velocity, $v_{\text {turb }} \leqslant 68 \mathrm{~m} / \mathrm{s}$ (Pagani et al. 2010a). This is lower than $v_{\text {crit }}$ for most of the possible collisions among DHGL populations. As an upper limit, we assume that $\Delta v=v_{\text {turb }}$, which leads to the shortest possible coagulation timescale, $\tau_{\text {coag. }}$ Following Stepnik et al. (2003), we define the coagulation timescale of one particle of species 1 onto one particle of species 2 as

$\tau_{\text {coag }}=\left[\pi\left(a_{1}+a_{2}\right)^{2} n_{1} \Delta v\right]^{-1}$,

where $n_{1}$ is the density of the species 1 . We define the average $\tau_{\text {coag }}$ between two species with size distributions $n\left(a_{1}\right)$ and $n\left(a_{2}\right)$, respectively, as

$\tau_{\text {coag }}^{-1}=\int_{a_{1}^{\min }}^{a_{1}^{\max }} n\left(a_{1}\right) d a_{1} \int_{a_{2}^{\min }}^{a_{2}^{\max }} n\left(a_{2}\right) \mathrm{d} a_{2} \pi\left(a_{1}+a_{2}\right)^{2} n_{1} \Delta v$.

This relation supposes that all the collisions lead to coagulation, which again puts a lower limit on $\tau_{\text {coag }}$. The corresponding values are given in Table 5 for cuts 1, 2, and 4.

However, the aggregates required to explain the emission and extinction profiles of L1506 contain more than two grains: $\langle a\rangle \sim 0.02 \mu \mathrm{m}$ for the aggregates with $10 \%$ of voids. According to Köhler et al. (2012), the coagulation of at least four big grains is required to increase the opacity at $250 \mu \mathrm{m}$ by a factor of $2-2.5$. We assume that the average aggregate is made of three aSil grains plus one LamC grain. As a first approximation, the coagulation timescale to stick three aSil grains together is supposed to be about twice the coagulation time of one aSil grain onto another aSil grain. Then, a LamC grain can stick onto this aSil aggregate or the aSil aggregate can stick onto a LamC grain. This leads to coagulation timescales of $\tau_{\mathrm{coag}} \sim 1.3-2.8 \times 10^{5} \mathrm{yrs}$ for cut $1, \tau_{\text {coag }} \sim 1.1-2.3 \times 10^{5}$ yrs for cut 2 , and $\tau_{\text {coag }} \sim$ $1.1-2.4 \times 10^{6} \mathrm{yrs}$ for cut 4 . The inclusion of small grains (PAHs and SamC) in the aggregates is very fast, so it can be neglected in our rough estimate of $\tau_{\text {coag. }}$.

Now that we have an estimate of $\tau_{\text {coag }}$, it can be compared to the cloud lifetime. This lifetime is at least equal to the free-fall timescale. According to Kawachi \& Hanawa (1998), the free-fall timescale of an isothermal cylinder with a constant density $n_{0}$ is

$\tau_{\mathrm{ff}}=\frac{1}{\sqrt{4 \pi G m_{\mathrm{H}} n_{0}}}$

where $G$ is the gravitational constant and the $m_{\mathrm{H}}$ the mass of the hydrogen atom. Considering the averaged density of our best-fitting models, $\langle n\rangle_{1} \sim 11000,\langle n\rangle_{2} \sim 7000$, and $\langle n\rangle_{4} \sim 6500 \mathrm{H} / \mathrm{cm}^{3}$, we get $\tau_{\text {coag }} \sim 2.6 \times 10^{5}, 3.2 \times 10^{5}$, and $3.3 \times 10^{5}$ yrs for cuts 1,2 , and 4 , respectively. Thus, the coagulation and free-fall timescales are of the same order. Because these free-fall timescales are lower limits for the cloud lifetime since we neglected any support, which would be able to slow down the gravitational collapse (e.g., the magnetic field or the rotation of the filament around its axis of symmetry), our results support the idea of a grain coagulation taking place inside the filament.

\subsection{Stability of the filament}

Pagani et al. (2010a) showed that the velocity profiles of $\mathrm{C}^{18} \mathrm{O}$, and $\mathrm{N}_{2} \mathrm{H}^{+}$prove an inward motion of the gas of at least $0.1 \mathrm{~km} \mathrm{~s}^{-1}$ in L1506C. They interpreted this as a proof of the collapse of the core. We can further test this finding by computing the masses per unit length of our best-fitting models:

$$
\begin{aligned}
& M_{\text {line }}=\int_{0}^{R_{\text {out }}} 2 \pi n_{\mathrm{H}}(r) r \mathrm{~d} r \\
& M_{\text {line }}=\pi n_{\mathrm{C}} R_{\text {flat }}^{2} \ln \left[1+\left(\frac{R_{\text {out }}}{R_{\text {flat }}}\right)^{2}\right] .
\end{aligned}
$$

This leads to $M_{\text {line }}=18.3_{-0.8}^{+1.7}, 14.2_{-0.7}^{+0.2}$, and $14.1 \pm 0.4 M_{\odot} / \mathrm{pc}$ for cuts 1,2 , and 4 , respectively. These linear masses can be compared to the critical mass defined by Ostriker (1964), above which the cloud collapses: $M_{\text {crit }}=2 c_{\mathrm{S}}^{2} / G$, where $c_{\mathrm{S}}$ is the sound speed and depends on the gas temperature. Following the prescription of Pagani et al. (2010a), we assume a gas temperature 
of $10 \mathrm{~K}$, which gives $M_{\text {crit }}=16.8 M_{\odot} / \mathrm{pc}$. Our results agree with the results of Pagani et al. (2010a), who found that the filament may be collapsing at the location of the first cut or L1506C $\left(M_{\text {line }} \gtrsim M_{\text {crit }}\right)$. At the two other locations along L1506, the linear masses are slightly lower. As we lack information on the gas temperature for cuts 2 and 4 and as the equilibrium state of a cloud depends on many parameters (external pressure, turbulence, and magnetic field), we cannot conclude on the stability of the filament at these locations.

\subsection{Main results and caveats}

With the advantage of the improved sensitivity, resolution, and spectral coverage from the Herschel PACS and SPIRE instruments, we studied the variations in the dust properties between the diffuse ISM and the centre of the L1506 cloud. To do so, we tested the data against the dust models described in Compiègne et al. (2011) and Ysard et al. (2012). Our subsequent findings are the following:

(i) the emission and extinction profiles of L1506 cannot be explained with dust grains similar to those found in the diffuse ISM;

(ii) they cannot be explained by a model where we consider that all the grains have coagulated to form compact or fluffy aggregates;

(iii) they can be reproduced by considering that the grains are of diffuse ISM type in the outer layers of the filament, whereas aggregates dominate the dust abundance above a certain density threshold (typically a few $10^{3} \mathrm{H} / \mathrm{cm}^{3}$ ).

Considering the first point, we tested the data against two different diffuse dust models (Draine \& Li 2007; Compiègne et al. 2011) and for several filament inclination angles. The results were unchanged. It was impossible to fit the emission profiles and the extinction was systematically overestimated. This also matches many previous studies that used various techniques to reach this result (see references in Sect. 1). Thus, we consider it is a definitive result that the emission and extinction in L1506 cannot be explained with grains of the diffuse ISM type.

At first, the second point appears more questionable. Indeed, we find that the far-IR emission can be easily explained with a single aggregate population, whereas the extinction is underestimated (for all the inclination angles tested, $0^{\circ} \leqslant \alpha \leqslant 40^{\circ}$ ). This result may come from our aggregate model. The question is: can the data be explained by an aggregate model with a different near-IR to submm opacity ratio? In Fig. 2, we show three different "evolved grain" models. The opacity of the dust model of Weingartner \& Draine (2001) at $250 \mu \mathrm{m}$ does not seem appropriate in explaining the data, as it is of the order of the opacity of the dust in the diffuse ISM. The aggregate models of Ormel et al. (2011) and Ossenkopf \& Henning (1994) have near- and far-IR opacities that are similar to those of our model (Ysard et al. 2012): their far-IR opacity differs from ours by $\sim 30 \%$ and in the visible and near-IR by $\sim 15 \%$. This is far from reaching the factors of 1.5 to 2.5 reported in Sect. 6.2 and by which our bestfitting models underestimate the extinction. These models may not represent the entire plausible range of dust models; however, it is an indication of the robustness of our result. Detailed studies for more molecular clouds, which undergo various environmental conditions, are required to strengthen the fact that no single dust model can simultaneously fit the dust emission and extinction (i.e., a variation in dust properties along the line-ofsight is required to simultaneously fit both the emission and the extinction).
If one trusts the robustness of the previous result, the third point comes naturally: diffuse ISM-type grains are found at the edge of the cloud and aggregates at the centre. This result is strengthened by the correspondence between our results and the modelling of the molecular gas lines performed by Pagani et al. (2010a). However, the exact numerical values (e.g., Table 3), especially the threshold density above which the aggregates dominate the dust abundance, are model-dependent. For instance, the simple addition of an ice mantle on top of the aggregates may lead to significantly different near-IR to far-IR opacity ratios, as can be seen in Fig. 2 .

This study made us aware of an intrinsic caveat of our aggregate model. As shown in Sect. 6.3.3, our aggregate opacity spectral index is too low in the far-IR and the submillimetre with $\beta \sim 1.3$. Because of this low value, we are not able to find any satisfactory fit for the $500 \mu \mathrm{m}$ emission profile of the third cut, and the best-fitting models for the three other cuts always lead to higher estimates of this same profile. This low $\beta$-value is due to the wavelength dependence of the absorption efficiency $\left(Q_{\mathrm{abs}}\right)$ of the carbon grains present in the DHGL model. Performing Mie calculations, Compiègne et al. (2011) defined their absorption and extinction efficiencies from the BE sample complex refractive index data derived from laboratory measurements by Zubko et al. (1996). This sample was assumed to be a good analogous to interstellar (hydrogenated) amorphous carbon. However, it appears that this experimental sample might not be the most appropriate to account for the interstellar carbonaceous dust.

\section{Conclusions}

We performed detailed modelling of the dust content and density structure of L1506, a dense filament in the Taurus molecular complex, and included full-radiative transfer calculations. We definitively excluded the possibility that the emission and extinction profiles of this filament could be explained using the properties of the dust found in the standard diffuse ISM. We showed that the dust far-IR opacity has to increase from the outer to the inner parts of L1506. We interpret this increase in the opacity as the formation of large aggregates. To fit the far-IR dust emission and extinction profiles simultaneously along the filament, aggregates with an average size in terms of mass of about $0.4 \mu \mathrm{m}$ and an increase in the opacity at $250 \mu \mathrm{m}$ of 1.8 to 2.2 are required. They have to prevail over diffuse ISM-type grains, when the local gas density reaches a few $1000 \mathrm{H} / \mathrm{cm}^{3}$. The exact numerical value of this threshold naturally depends on the dust model chosen to fit the data. The size of these aggregates is not far from what is required to explain the coreshine observed in molecular clouds (Pagani et al. 2010b; Steinacker et al. 2010). Using a simple approach, we showed that these aggregates may have time to form inside the filament within the cloud lifetime. Our best-fitting models finally reveal that the width of the filament varies according to the column density. More modelling of dense filaments is needed to confirm our results. An important breakthrough may thus arise from detailed modelling including both the emitted and scattered light in addition to the extinction.

Acknowledgements. N.Y. acknowledges the support of a CNES post-doctoral research grant. We thank our anonymous referee, whose useful comments helped us to improve the content of this paper. SPIRE has been developed by a consortium of institutes led by Cardiff Univ. (UK) and including: Univ. Lethbridge (Canada); NAOC (China); CEA, LAM (France); IFSI, Univ. Padua (Italy); IAC (Spain); Stockholm Observatory (Sweden); Imperial College London, RAL, UCL-MSSL, UKATC, Univ. Sussex (UK); and Caltech, JPL, NHSC, Univ. Colorado (USA). This development has been supported by national funding agencies: CSA (Canada); NAOC (China); CEA, CNES, CNRS (France); ASI (Italy); MCINN (Spain); SNSB (Sweden); STFC, UKSA (UK); and NASA (USA). 


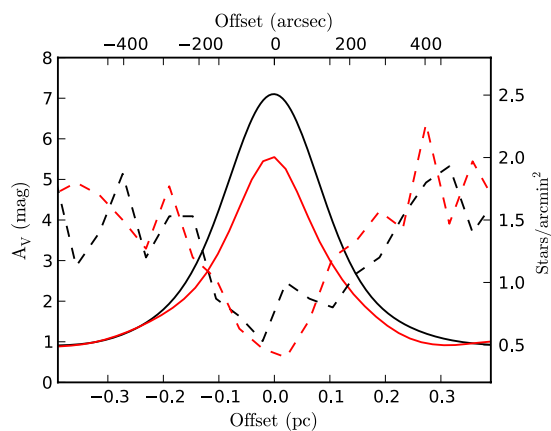

Fig. A.1. Simulation of possible bias in NICER estimates. The black solid curve shows the assumed true $A_{V}$ profile, convolved to a resolution of $200^{\prime \prime}$. The red solid line shows the extinction profile recovered with NICER algorithm from the simulated observations. The dashed lines and the right hand scale indicate the stellar density in the case of the first cut (black dashed line) and in the simulation (red dashed line).

\section{Appendix A: Estimated bias of extinction maps}

The resolution of the NICER extinction maps is $200^{\prime \prime}$. This is not much smaller than the width of the filaments and can bias the estimates of peak extinction because more background stars are visible through the filament edges where the column density is lower. To estimate the magnitude of the error, we analysed a set of simulated observations. We started with the observed $A_{V}$ profile of the first cut and used that to construct a two-dimensional image of a filament with the same extinction profile. We took 2MASS stars observed towards a nearby lowextinction field and distributed them over the image with a stellar density that is similar to the actual Taurus observations. The signal of the background stars was attenuated and the photometric errors were increased to correspond to the noise levels and the detection thresholds of the Taurus field. The modified magnitudes were fed to the NICER routine to construct an "observed" extinction map.

Figure A.1 shows as a solid black line the input $A_{V}$ profile convolved to the resolution of $200^{\prime \prime}$, which has a maximum of $\sim 7^{\mathrm{m}}$. The red line shows the $A_{V}$ profile recovered from the calculated NICER map, which has a maximum of $\sim 5.5^{\mathrm{m}}$. The noise is low because we have averaged data over wider strips across the simulated filament. The peak extinction is underestimated by $\sim 21 \%$, while the contrast between the filament and the assumed background extinction is lower by $\sim 25 \%$. The recovered peak extinction of $\sim 5.5^{\mathrm{m}}$ magnitudes for an angular resolution of $200^{\prime \prime}$ is significantly lower than the assumed true peak value of $\sim 9^{\mathrm{m}}$ magnitudes at full angular resolution. This is in roughly equal parts due to the bias and due to the natural effect of a change in the spatial resolution. This also means that the simulation is only a conservative estimate of the true bias. If we repeat this simulation for a filament with a lower visual extinction $\left(A_{V}=5^{\mathrm{m}}\right.$ at $200^{\prime \prime}$ angular resolution), the recovered peak extinction is still underestimated by $16 \%$.

\section{References}

Abergel, A., Boulanger, F., Mizuno, A., \& Fukui, Y. 1994, ApJ, 423, L59 Abergel, A., Bernard, J. P., Boulanger, F., et al. 1996, A\&A, 315, L329 Abergel, A., Arab, H., Compiègne, M., et al. 2010, A\&A, 518, L96 André, P., Men'shchikov, A., Bontemps, S., et al. 2010, A\&A, 518, L102 Arzoumanian, D., André, P., Didelon, P., et al. 2011, A\&A, 529, L6 Bazell, D., \& Dwek, E. 1990, ApJ, 360, 142
Bergin, E. A., \& Tafalla, M. 2007, ARA\&A, 45, 339

Bernard, J. P., Abergel, A., Ristorcelli, I., et al. 1999, A\&A, 347, 640

Bohren, C. F., \& Huffman, D. R. 1983, Absorption and scattering of light by small particles (New York: Wiley)

Cambrésy, L., Boulanger, F., Lagache, G., \& Stepnik, B. 2001, A\&A, 375, 999

Cardelli, J. A., Clayton, G. C., \& Mathis, J. S. 1989, ApJ, 345, 245

Compiègne, M., Verstraete, L., Jones, A., et al. 2011, A\&A, 525, A103

Dominik, C., \& Tielens, A. G. G. M. 1997, ApJ, 480, 647

Dorschner, J., \& Henning, T. 1995, A\&ARv, 6, 271

Draine, B. T. 2003, ARA\&A, 41, 241

Draine, B. T., \& Anderson, N. 1985, ApJ, 292, 494

Draine, B. T., \& Li, A. 2007, ApJ, 657, 810

Fischera, J., \& Martin, P. G. 2012a, A\&A, 547, A86

Fischera, J., \& Martin, P. G. 2012b, A\&A, 542, A77

Fitzpatrick, E. L. 1999, PASP, 111, 63

Flagey, N., Noriega-Crespo, A., Boulanger, F., et al. 2009, ApJ, 701, 1450

Goldsmith, P. F., Heyer, M., Narayanan, G., et al. 2008, ApJ, 680, 428

Griffin, M. J., Abergel, A., Abreu, A., et al. 2010, A\&A, 518, L3

Hartmann, L. 2002, ApJ, 578, 914

Heitsch, F. 2013, ApJ, 769, 115

Hill, T., Motte, F., Didelon, P., et al. 2011, A\&A, 533, A94

Juvela, M. 2005, A\&A, 440, 531

Juvela, M., \& Ysard, N. 2011, ApJ, 739, 63

Juvela, M., Pelkonen, V.-M., Padoan, P., \& Mattila, K. 2008, A\&A, 480, 445

Juvela, M., Ristorcelli, I., Pelkonen, V.-M., et al. 2011, A\&A, 527, A111

Juvela, M., Ristorcelli, I., Pagani, L., et al. 2012, A\&A, 541, A12

Kawachi, T., \& Hanawa, T. 1998, PASJ, 50, 577

Köhler, M., Guillet, V., \& Jones, A. 2011, A\&A, 528, A96

Köhler, M., Stepnik, B., Jones, A. P., et al. 2012, A\&A, 548, A61

Kramer, C., Richer, J., Mookerjea, B., Alves, J., \& Lada, C. 2003, A\&A, 399 1073

Laureijs, R. J., Clark, F. O., \& Prusti, T. 1991, ApJ, 372, 185

Li, A., \& Draine, B. T. 2001, ApJ, 554, 778

Lombardi, M., \& Alves, J. 2001, A\&A, 377, 1023

Martin, P. G., Roy, A., Bontemps, S., et al. 2012, ApJ, 751, 28

Men'shchikov, A., André, P., Didelon, P., et al. 2010, A\&A, 518, L103

Nguyen L., Q., Motte, F., Hennemann, M., et al. 2011, A\&A, 535, A76

Nielbock, M., Launhardt, R., Steinacker, J., et al. 2012, A\&A, 547, A11

Ormel, C. W., \& Cuzzi, J. N. 2007, A\&A, 466, 413

Ormel, C. W., Paszun, D., Dominik, C., \& Tielens, A. G. G. M. 2009, A\&A, 502, 845

Ormel, C. W., Min, M., Tielens, A. G. G. M., Dominik, C., \& Paszun, D. 2011, A\&A, 532, A43

Ossenkopf, V. 1993, A\&A, 280, 617

Ossenkopf, V., \& Henning, T. 1994, A\&A, 291, 943

Ostriker, J. 1964, ApJ, 140, 1056

Pagani, L., Ristorcelli, I., Boudet, N., et al. 2010a, A\&A, 512, A3

Pagani, L., Steinacker, J., Bacmann, A., Stutz, A., \& Henning, T. 2010b, Science, 329, 1622

Palmeirim, P., André, P., Kirk, J., et al. 2013, A\&A, 550, A38

Patanchon, G., Ade, P. A. R., Bock, J. J., et al. 2008, ApJ, 681, 708

Pilbratt, G. L., Riedinger, J. R., Passvogel, T., et al. 2010, A\&A, 518, L1

Planck Collaboration 2011a, A\&A, 536, A25

Planck Collaboration 2011b, A\&A, 536, A24

Planck Collaboration 2011c, A\&A, 536, A19

Planck Collaboration 2011d, A\&A, 536, A23

Poglitsch, A., Waelkens, C., Geis, N., et al. 2010, A\&A, 518, L2

Roberts, J. F., Rawlings, J. M. C., Viti, S., \& Williams, D. A. 2007, MNRAS, 382, 733

Roy, A., Martin, P. G., Polychroni, D., et al. 2013, ApJ, 763, 55

Skrutskie, M., Cutri, R., Stiening, R., et al. 2006, AJ, 131, 1163

Steinacker, J., Pagani, L., Bacmann, A., \& Guieu, S. 2010, A\&A, 511, A9

Stepnik, B., Abergel, A., Bernard, J.-P., et al. 2003, A\&A, 398, 551

Stognienko, R., Henning, T., \& Ossenkopf, V. 1995, A\&A, 296, 797

Stutz, A., Launhardt, R., Linz, H., et al. 2010, A\&A, 518, L87

van Breemen, J. M., Min, M., Chiar, J. E., et al. 2011, A\&A, 526, A152

Ward-Thompson, D., Scott, P. F., Hills, R. E., \& Andre, P. 1994, MNRAS, 268, 276

Weingartner, J. C., \& Draine, B. T. 2001, ApJ, 548, 296

Whittet, D. C. B., Bode, M. F., Longmore, A. J., et al. 1988, MNRAS, 233, 321

Wright, E. L. 1987, ApJ, 320, 818

Ysard, N., Juvela, M., Demyk, K., et al. 2012, A\&A, 542, A21

Zhilkin, A. G., Pavlyuchenkov, Y. N., \& Zamozdra, S. N. 2009, Astron. Rep., 53,590

Zubko, V. G., Mennella, V., Colangeli, L., \& Bussoletti, E. 1996, MNRAS, 282, 1321 